\title{
Subjective confidence acts as an internal cost-benefit factor when choosing between tasks
}

\author{
Nomi Carlebach \& Nick Yeung \\ Department of Experimental Psychology, University of Oxford
}

\begin{abstract}
Author Note
Correspondence concerning this paper should be addressed to Nomi Carlebach, Department of Experimental Psychology, University of Oxford, Anna Watts Building, Oxford OX2 6GG, UK.

Email: naomi.carlebach@psy.ox.ac.uk
\end{abstract}

Acknowledgements

Nomi Carlebach was supported by a DPhil studentship from the Department of Experimental Psychology, University of Oxford, and grants from the British Friends of the Hebrew University, Funds for Women Graduates, B'nai B'rith Leo Baeck (London) Lodge and the Anglo-Israel Association. We thank Lucie Charles for her help with Matlab scripts. Data and analysis code can be freely accessed online through the Open Science Framework at https://osf.io/tu5y7/?view_only=f87a3390a5a9434c9d3e289517a285c6

Word count: 13,698 


\begin{abstract}
Upon making a decision, we typically have a sense of the likelihood that the decision we reached was a good one, i.e. a degree of confidence in our choice. In a series of five experiments, we tested the hypothesis that confidence acts as an intrinsic cost-benefit factor when choosing between tasks, biasing people towards situations in which they experience higher confidence. Participants performed a task-selection paradigm in which they chose on each trial between two perceptual-judgement tasks that were matched for objective difficulty but differed in participants' experienced confidence, with confidence manipulated via differences in the strength of post-decisional evidence. The results show that participants exhibited a preference for tasks in which they reported higher confidence. The effect of confidence on task selection went above and beyond simple error detection, with people not only avoiding tasks in which they believed they made an error, but also tending to select tasks in which they experienced higher confidence in their correct responses. Moreover, preference for high-confidence tasks was evident even when external feedback was provided on every trial. Collectively, these results indicate that subjective confidence guides choices of which situations and tasks to engage with, as a valuable indicator of likely success.
\end{abstract}

Keywords: confidence, metacognition, decision making, cognitive control 
Public Significance Statement: When making decisions, people usually have a sense of how likely it is that they have made the correct choice. The present study investigates how this sense of confidence influences subsequent decisions of which tasks to engage with. Our results demonstrate that people select situations in which they experience higher confidence more often than those in which confidence is lower. These results highlight the role of confidence in adaptive decision making in our daily lives, guiding us towards situations and tasks in which we are ultimately more likely to succeed. 


\section{Introduction}

In our daily lives, we are constantly faced with the need to make decisions. These can be significant decisions, such as where to buy a house, or smaller decisions, for example whether a fruit is ripe enough to eat. Upon making a decision, we typically have a sense of the likelihood that the decision we reached was a good one, i.e. a degree of confidence in our choice. Recent years have shown a surge of interest in decision confidence, with much research on the neural and computational basis of confidence judgments (Boldt \& Yeung, 2015; Fleming \& Daw, 2017; Fleming \& Frith, 2014; Kepecs, Uchida, Zariwala, \& Mainen, 2008; Kiani \& Shadlen, 2009; Sanders, Hangya, \& Kepecs, 2016; Yeung \& Summerfield, 2014). Studies have demonstrated that people can reliably detect errors in their performance (Rabbitt, 1966), and that subjective confidence strongly reflects objective accuracy (Baranski \& Petrusic, 1994). However, despite the evident connection between confidence and decision making, and our growing understanding of how confidence judgments are formed, their functional role and how they affect subsequent decisions has been much less thoroughly explored. The present research addresses this issue to consider one potential role of confidence in adaptive behavior: in guiding our choices of which situations and tasks to engage in.

Previous research has considered the role of decision confidence in adaptive behavior and learning as providing a subjective estimate of the probability that a decision was correct, information that can be particularly useful when external feedback is lacking. For example, it has been suggested that confidence can be used to enhance performance by guiding the amount of evidence required before reaching a decision (the decisional threshold), so that people gather more evidence before committing to a decision when confidence is low compared to when confidence is high (Yeung \& Summerfield, 2012). In multi-stage decisions, where achieving your goal requires success in all stages, confidence is carried over from one stage to the next and used to adjust the decisional threshold of subsequent stages (van den Berg, Zylberberg, 
Kiani, Shadlen, \& Wolpert, 2016). Recent empirical studies have also demonstrated the role of confidence in group decision-making, with groups outperforming individuals when they weight opinions according to the confidence with which they are expressed (Bahrami et al., 2010; Bang et al., 2014), and in information seeking, with participants selectively seeking further information before committing to a choice when they are initially low in confidence (Desender, Boldt, \& Yeung, 2018; Desender, Murphy, Boldt, Verguts, \& Yeung, 2019). Particularly relevant to the present study, it has been suggested that confidence can provide a 'common currency' that can allow different types of decisions to be compared and prioritized (de Gardelle, Le Corre, \& Mamassian, 2016; de Gardelle \& Mamassian, 2014).

Further insight into the possible functional role of confidence comes from studies on the function of other metacognitive judgments beyond decision confidence. Metacognition, broadly defined as "cognitions about cognitions" (Flavell, 1979), can be divided into metacognitive knowledge (what we know about cognition in general), and the online monitoring and control of our own cognitive processes (Nelson \& Narens, 1990). There is a notably rich corpus of findings from studies of metacognitive knowledge and monitoring as they relate to memory processes in learning and education (Bjork, Dunlosky, \& Kornell, 2013; Koriat, 2006). Within this field, research has demonstrated the important role of metacognitive judgments, such as feeling of knowing and judgments of learning, on successful self-regulated learning (Bjork et al., 2013). Of particular relevance here, this research has documented how metacognitive judgments not only influence immediate performance — such as an initial feeling of knowing determining whether or not to attempt memory retrieval (Koriat \& Levy-Sadot, 2001; Reder, 1987) — but also guide longer-term strategic control of behavior, such as decisions about what information to try to encode in memory, or indeed whether to attempt to learn at all (Murayama, Blake, Kerr, \& Castel, 2016; Nelson, Dunlosky, Graf, \& Narens, 1994). The present research applies a similar reasoning to decision confidence, specifically exploring its 
role beyond the scope of an immediate decision ("am I confident enough to make this decision now?") to include strategic choices about which situations to pursue ("am I confident enough in this task to engage with it?").

In this way, our study draws research on decision confidence together with a hitherto separate line of work exploring how people choose between different tasks or lines of action. In this other work, a core hypothesis is that adaptive selection is guided by the learnt potential costs and benefits of each line of action. These costs and benefits include the outcomes of our actions, such as monetary loss or gain (Kahneman \& Tversky, 1984), as well as costs and benefits intrinsic to the action itself. For example, a well-established intrinsic cost of an action is the amount of physical work or effort that it entails (Hull, 1943; Solomon, 1948; Walton, Kennerley, Bannerman, Phillips, \& Rushworth, 2006). This concept has been extended to cognitive as well as physical effort (Apps, Grima, Manohar, \& Husain, 2015; Kool, McGuire, Rosen, \& Botvinick, 2010; Westbrook, Lamichhane, \& Braver, 2019). In particular, in a set of experiments that provided the methodological basis for the present experiments, Kool and colleagues employed a demand selection task in which participants chose between cues associated with tasks requiring different degrees of cognitive effort. The participants were not instructed about these associations, but could learn them through experience of the outcomes of their cue selections. Kool et al.'s results demonstrate that people tend to avoid selecting the line of action requiring more mental effort (Kool et al., 2010). Interestingly, although in this study they report that participants were unaware of the experimental manipulation, a later study found bias towards the less demanding task only when participants noticed the differences in effort demands (Gold et al., 2015). This finding raises questions regarding the role that perceived as opposed to objective costs and benefits play in the development of task preferences. 
Two recent studies addressing this issue have found evidence for the involvement of metacognitive awareness in task selection. Dunn and colleagues found that even when objective performance is equal, participants avoid situations which they believed would be more effortful, time demanding and less accurate (Dunn, Lutes, \& Risko, 2016). Similarly, in another study that manipulated cognitive demand using subliminal priming, bias towards a low-demand context was only apparent in participants who reported differences in task difficulty (Desender, Calderon, Van Opstal, \& Van den Bussche, 2017). Based on the results described above, it seems plausible that confidence could affect task selection in a fashion similar to related metacognitive judgements of effort and difficulty. There would be theoretical value in showing this relationship: Whereas there is ongoing debate about what exactly constitutes cognitive effort and what factors lead to its avoidance (Shenhav et al., 2017), decision confidence is well defined - as the internal belief that a response made was correctand computationally well specified — as a transformation of the evidence accumulated in favor of a decision (even if there is debate about the precise detail of this transformation; Pleskac \& Busemeyer, 2010; Pouget, Drugowitsch, \& Kepecs, 2016; Sanders et al., 2016). Thus, linking confidence to task choice would have meaningful theoretical implications through establishing a clear computational basis of task preferences.

Following this rationale, we hypothesized that people's confidence in their decisions acts as a cost or benefit factor intrinsic to the decision itself (cf. Lebreton, Abitbol, Daunizeau, \& Pessiglione, 2015). If this hypothesis is true, confidence should affect task selection even when controlling for other factors such as objective performance or external rewards. Importantly, we expected confidence to influence behavior in a graded fashion, beyond the binary classification of a response as being correct or incorrect. Specifically, we expected people to demonstrate a bias towards tasks in which they were more confident, even if they detected similar numbers of errors across the different tasks. As such, our predictions differ 
from those expected based solely on error aversion or classic reinforcement learning theories, whereby behavior is adjusted based on the outcome (i.e. accuracy or reward) of an action (Holroyd \& Coles, 2002; Holroyd, Yeung, Coles, \& Cohen, 2005). Extending this idea, we asked whether confidence will influence behavior even when external feedback is present, so that participants can know objectively whether or not their responses were correct without having to rely on their own internal evaluations. If effects of confidence are apparent even in the presence of feedback, this result would support the view that the fine-grained confidence signal is integrated into decisions automatically (Lebreton et al., 2015), and not only used as an internal monitoring signal when external feedback is lacking.

We tested these predictions in a series of five experiments, using a task-selection paradigm in which participants chose freely between two cues that (initially unbeknownst to them) were associated with decisions of differing levels of confidence. Specifically, participants performed a perceptual decision task in which they reported which of two boxes contained more dots, with the number of dots in each box changing dynamically during stimulus presentation, both before and after the decision (Charles \& Yeung, 2019). Regardless of the cue chosen, participants encountered a decision of equivalent difficulty (i.e., the mean dot difference between the two boxes was identical for both cues). However, after participants registered their decision, the stimulus continued to dynamically evolve, with the strength of post-decisional evidence varying according to the selected cue. This manipulation allowed us to break the typical link between subjective confidence and the initial difficulty and accuracy of decisions (Kepecs et al., 2008; Kiani \& Shadlen, 2009), in line with recent theoretical models (Moran, Teodorescu, \& Usher, 2015; Pleskac \& Busemeyer, 2010; Yu, Pleskac, \& Zeigenfuse, 2015) and neurophysiological evidence (Boldt \& Yeung, 2015; Fleming, Van Der Putten, \& Daw, 2018; Murphy, Robertson, Harty, \& O’Connell, 2015) suggesting that confidence incorporates information that is accumulated after the initial decision has been made. We 
predicted that differences in post-decisional evidence strength - either in favor of or against the initial choice-would lead to differences in the confidence levels associated with each cue, and that this in turn would lead to the development of a bias towards tasks associated with higher confidence, even across tasks matched for objective decision difficulty (as reflected in accuracy and reaction time). To anticipate, our results support this hypothesis, with people selecting more often the task in which they experience and report higher confidence.

\section{General Methods}

\section{Task and procedure}

All five experiments shared the same general method, comprising 4 blocks of a task selection paradigm with 80 trials per block. Each trial was divided into three sections: Task selection, perceptual judgment task, and confidence rating (see Figure 1). During task selection, participants chose one of two cues associated with perceptual judgment tasks that were identical except for the strength of post-decisional evidence presented. Participants were instructed to choose between the cues freely, and told that if one began to feel preferable they could choose it more often. They were not told the nature of the differences between the tasks associated with the cues. Trials started with a grey fixation cross presented in the center of the screen. After $300 \mathrm{~ms}$, the cues (different colored circles) appeared symmetrically above and 


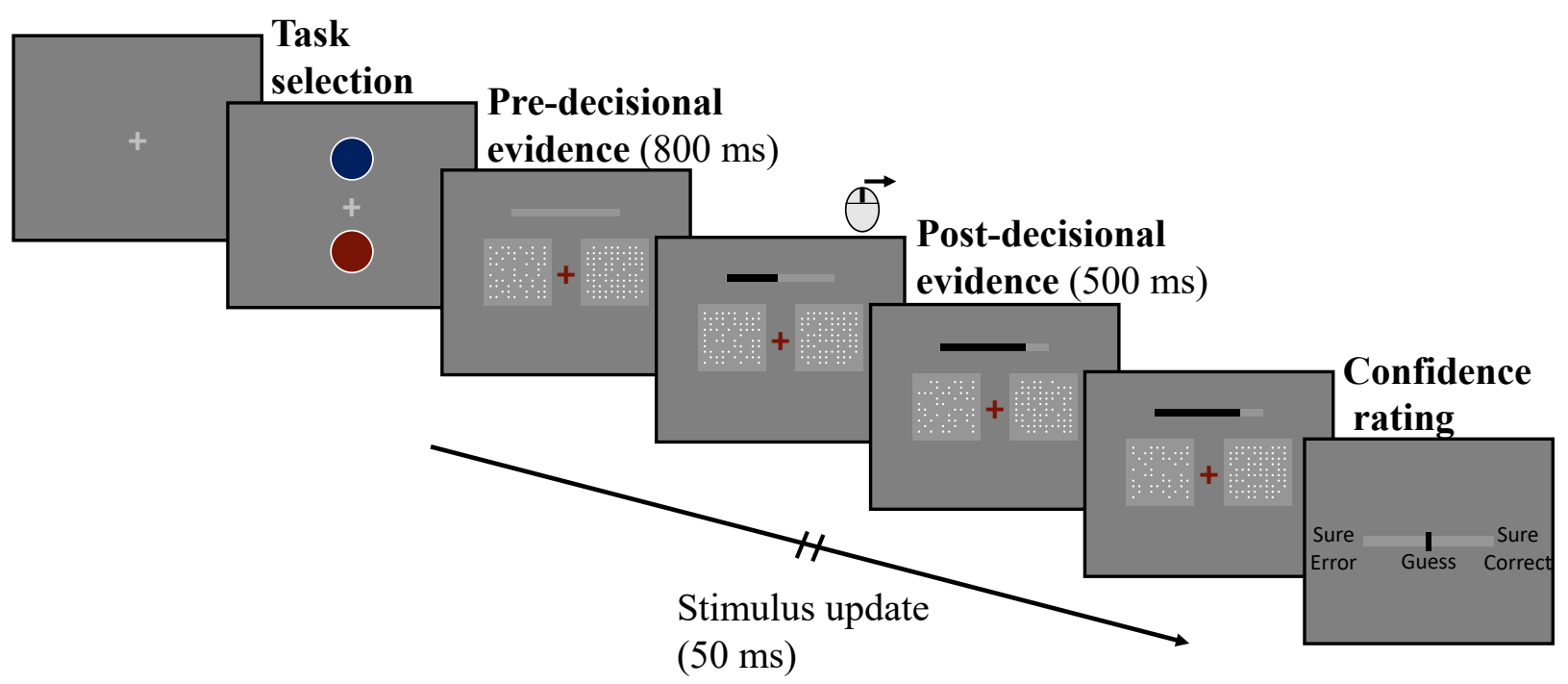

Figure 1. Task Design. Trials began with a choice between task cues associated with different post-decisional evidence. Following task choice, participants made a speeded judgment about which of two squares contained more dots on average. The number of dots was sampled from two normal distributions centered around different means, and was updated every $50 \mathrm{~ms}$. Dot stimuli remained on the screen and continued to be updated every $50 \mathrm{~ms}$ after participants' responses. After viewing post-decisional evidence, participants rated their confidence in their response.

below the fixation cross. 8 circles in different colors (red, green, blue, yellow, orange, purple, pink and cyan) were used throughout the experiment, with two colors randomly selected for each block, and assigned randomly to one of two locations in which they appeared throughout the entire block. Participants had unlimited time to select a cue using the mouse cursor. $200 \mathrm{~ms}$ after cue selection, the fixation cross changed color to that of the selected cue, and after another $500 \mathrm{~ms}$ the fixation cross flashed, indicating the perceptual task would start shortly. Following a varying delay of 101-500 ms, two empty grey squares appeared on either side of the fixation for $300 \mathrm{~ms}$, after which dots appeared within the squares and participants were asked to judge which square contained more dots on average. The number of dots and their locations within the squares were updated every $50 \mathrm{~ms}$, with dot numbers sampled randomly from two distributions with different means (see specific experiments) and an equal standard deviation 
of 20 dots. Thus, although the number of dots within each square changed during the trial, the correct square contained more dots on average. The correct square (left vs. right) was randomized across trials, with each square being correct on half of the trials in each block. Participants had up to $800 \mathrm{~ms}$ to respond by pressing the right or left mouse button, with a bar at the top of the screen indicating time progression. After participants responded, the stimulus remained onscreen and continued to be updated every $50 \mathrm{~ms}$ for an additional $500 \mathrm{~ms}$, allowing the accumulation of additional post-decisional evidence. This specific period was chosen based on previous indications that evidence presented during this time influenced confidence ratings (Charles \& Yeung, 2019). Importantly, the distances between the dot number distributions were changed in this post-decisional period, bringing the mean difference between boxes either further apart or closer together. Tasks associated with each cue differed in the strength of postdecisional evidence presented (i.e. the mean difference in dot numbers after the participant's response), allowing the manipulation of participants' confidence. The exact manipulation of post decisional evidence varied across experiments, and is detailed in the corresponding methods sections below.

In the final part of each trial, participants were asked to rate their confidence in their response on a 50-point scale ranging from "sure incorrect" (low confidence) to "sure correct" (high confidence), with "guess" in the center. The starting location of the cursor on this scale varied randomly across trials, and participants responded by moving the cursor to the appropriate location and clicking the left mouse button. There was no time limit for this part. If participants did not respond to the dot task within the $800 \mathrm{~ms}$ time limit, the trial ended and the next trial began, with the post-decisional evidence and confidence sections skipped.

Before the main experiment began, participants were given written instructions and trained on the dot task. Participants were required to answer a minimum of 12 out of 20 trials $(60 \%)$ correctly in the training block, and training was repeated until they reached this 
requirement. During the training block, visual feedback on the first-order judgment was given after every trial ("correct", "incorrect", "too slow") to assist participants in learning the task. Except for Experiment 4, feedback was not given during the main experimental blocks. However, participants were shown the percentage of correct first-order judgments they made at the end of every block to help keep them engaged. After completing the computer task participants answered the "need for cognition" scale (Petty, Cacioppo \& Kao, 1984) and a debriefing questionnaire assessing their awareness of the experimental manipulation (adapted from Kool et al., 2010). Findings relating to these questionnaire measures are presented in combination for all 5 experiments below in the final section of results. All procedures were approved by the Oxford University Medical Sciences Interdivisional Research Ethics Committee.

\section{Stimuli and Apparatus.}

All stimuli were created and presented in Matlab using Psychtoolbox3 extension (Brainard, 1997; Kleiner et al., 2007; Pelli, 1997). Stimuli were presented on a 20-inch CRT screen with grey background $(128,128,128)$, a refresh rate of $60 \mathrm{~Hz}$, and $1600 \mathrm{x} 1200$ resolution. The task cues were circles in 8 different colors (red, green, blue, yellow, orange, purple, pink and cyan). Dot stimuli were comprised of two grey squares $(150,150,150)$, in which white dots were presented $(200,200,200)$. The experiments took place in a dimly lit room, with participants sitting at a comfortable viewing distance from the screen.

\section{Experiment 1}

Experiment 1 aimed to test our main hypothesis: that confidence holds an intrinsic value in the decision-making process, so that people should prefer situations in which they are more confident (even when controlling for the difficulty of the first-order decision). Participants 
chose between two cues and performed a task associated with the chosen cue. To manipulate participants' confidence independent of task difficulty, cues were associated with tasks that were identical in the pre-decision stage, but which differed in terms of post-decisional evidence. One cue was associated with a task in which the evidence supporting the participant's decision was boosted after their decision, which should lead to relatively high confidence (Charles \& Yeung, 2019; Fleming et al., 2018). The other cue was associated with a task in which the evidence supporting the decision was reduced, which should lead to lower confidence. We predicted that participants would select the cue associated with a boost in evidence supporting their choice (and hence with higher confidence) more often than the alternative cue.

\section{Methods}

Participants. 20 participants took part in the experiment (14 females, ages 19-35 years, mean age $24.5, S D=3.49$ ). All participants had normal or corrected to normal vision. They provided written informed consent and were compensated with course credit or payment for their participation. To select an appropriate sample size and ensure that the current task selection paradigm would be able to capture task preferences, we ran a pilot study. The sample size for the pilot $(n=20)$ was chosen on the basis of previous experiments that have found effects of subjective confidence on decision making (specifically on information sampling) using this number of participants (Desender et al., 2018). In the pilot, each cue was associated with a different objective difficulty of the dot task (i.e., also varied in dot difference prior to participants' decisions). One cue was associated with strong evidence, with the distributions of dot numbers centered around $M_{C}=222, M_{I}=178$ (mean dot numbers in the correct and incorrect boxes, respectively). The second cue was associated with weak evidence, with distributions of dot numbers that were closer together, centered around $M_{C}=212, M_{I}=188$. 
The dot number distributions were identical for the pre- and post-decision stages. Under these conditions, we found that participants showed a consistent preference towards the easier, strong-evidence task than the more difficult weak-evidence task, with a large effect size (mean proportion of easy task selections $=0.63, t(19)=4.17$, Cohen's $\left.d_{z}=0.93\right)$. Though we did not expect our post-decisional manipulation to produce an effect size as large as this, with a sample size of 20 we would be able to detect an effect that is $2 / 3$ this size $\left(d_{\mathrm{z}}=0.62\right)$ with a power of .85. Power calculations were performed using the GPower3 program (Faul, Erdfelder, Lang, \& Buchner, 2007).

Stimuli and Procedure. The experiment followed the general procedure described above, with participants asked to choose on each trial between cues that were associated with subtly different perceptual decision tasks. Thus, regardless of participants' choice of cue, the difficulty of the perceptual task was the same on all trials, with fixed evidence strength in the pre-decision stage $\left(M_{C}=212, M_{I}=188\right)$. However, after participants selected the box that they thought contained more dots, post-decisional evidence differed according to the cue chosen at the start of the trial. In the boost selected box condition, 10 dots were added to the mean number of dots in the chosen box, and 10 dots removed from the unchosen box, with the stimulus continuing to update every $50 \mathrm{~ms}$, as in the pre-decision stage. Thus, for this cue, strong evidence was presented following correct responses $\left(M_{C}=222, M_{I}=178\right)$ and weak evidence was presented following incorrect responses $\left(M_{C}=202, M_{I}=198\right)$. We expected this manipulation to increase participants' confidence (i.e., higher subjective probability that the response was correct) regardless of the accuracy of the initial decision-with strong evidence reinforcing correct choices, and weak evidence making errors less likely to be detected. In the boost alternative box condition, on the other hand, 10 dots were removed from the mean of the chosen box and 10 dots added to the unchosen box after the decision. Thus, weak evidence was presented following correct responses $\left(M_{C}=202, M_{I}=198\right)$ and strong 
evidence was presented following incorrect responses $\left(M_{C}=222, M_{I}=178\right)$, which should reduce confidence by reducing the strength of evidence in favor of initially-correct responses and increasing the likelihood that errors would be detected. Note that the underlying mean continued to favor the correct box in the post-decisional period for both conditions.

\section{Results}

Manipulation effects on performance and confidence. Since the two tasks were identical, by design, in terms of average strength of evidence presented prior to participants' first-order judgment, performance in the tasks was not expected to differ. Indeed, performance levels were similar, with participants responding to a similar proportion of trials within time limits $(M=.95 S D=.04 \mathrm{vs}, M=.95, S D=.03$ for boost selected and boost alternative tasks; $t$ $<1)$. On trials with on-time responses, similar accuracy levels were observed for the boost selected $(M=0.74, S D=0.09)$ compared to boost alternative task $(M=0.73, S D=0.08)$, with no consistent differences across participants $\left(t(19)=1.08, p=.30, d_{z}=0.24\right)$. Similarly, mean reaction times were well matched across tasks $(M=598 \mathrm{~ms}, S D=42$ vs $M=597 \mathrm{~ms}, S D=46$, for the boost selected and boost alternative tasks, respectively; $t<1$ ). Nevertheless, manipulation of post-decisional evidence had the hypothesized effect on confidence ratings, as revealed in a 2 x 2 repeated measures ANOVA with factors of task (boost selected box, boost alternative box) and decision accuracy (correct, incorrect; see Figure 2A for the results and Table 1 for data on trial numbers). A significant main effect of accuracy indicated that, as 

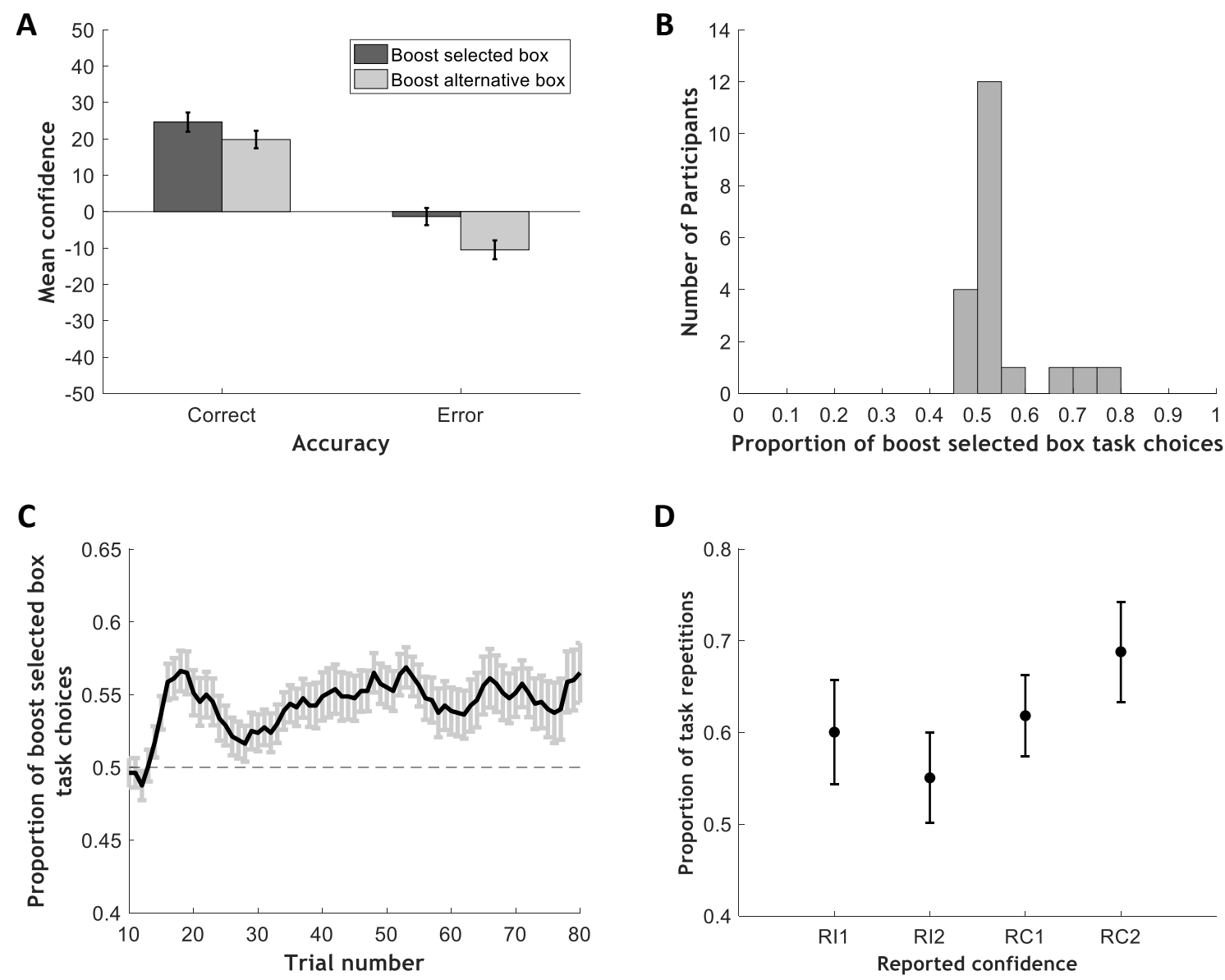

Figure 2. Results of Experiment 1. A, Effects of post-decisional evidence manipulation on confidence. Mean confidence ratings for the boost selected box and boost alternative box tasks, divided according to trial accuracy. B, Distribution of individual participants' proportion of boost selected box task choices $(n=20)$. $C$, Ten-trial running average showing the proportion of boost selected box task choices across blocks. Dashed line represents chance level. $\boldsymbol{D}$, Mean proportion of task repetitions according to reported confidence. Trials were first divided into groups according to reported accuracy $(\mathrm{RI}=$ reported incorrect, $\mathrm{RC}=$ reported correct), followed by a division according to median splits within the accuracy group ( 1 = below median confidence, 2 = above median confidence). Note: Error bas represent standard errors of the mean (SEMs).

expected, participants were more confident in their correct responses than their errors $(F(1,19)$ $\left.=90.60, p<.001, \eta_{p}^{2}=.83\right)$. Crucially, a significant main effect was also found for task $(F(1$, 19) $=45.77, p<.001, \eta_{p}^{2}=.71$ ), with higher confidence ratings in the boost selected task than in the boost alternative task. The interaction between confidence and accuracy was also significant $\left(F(1,19)=5.41, p<.05, \eta_{p}^{2}=.22\right)$, reflecting a greater confidence difference between tasks on error trials. 


\begin{tabular}{ccccccccccccccccc} 
& \multicolumn{1}{c}{} & \multicolumn{1}{c}{ Correct } & \multicolumn{1}{c}{ Error } & \multicolumn{4}{c}{ Correct } & \multicolumn{3}{c}{ Error } \\
\cline { 2 - 7 } Exp. & $M$ & Min & Max & $S D$ & $M$ & Min & Max & $S D$ & $M$ & Min & Max & SD & $M$ & Min & Max & $S D$ \\
\hline 1 & 122 & 87 & 187 & 25 & 43 & 17 & 71 & 14 & 102 & 55 & 130 & 20 & 39 & 11 & 63 & 14 \\
2 & 120 & 78 & 177 & 24 & 46 & 22 & 84 & 14 & 103 & 41 & 125 & 22 & 38 & 18 & 60 & 12 \\
3 & 143 & 80 & 249 & 45 & 47 & 19 & 72 & 14 & 85 & 8 & 158 & 38 & 32 & 2 & 57 & 15 \\
4 & 129 & 79 & 196 & 26 & 37 & 21 & 56 & 9 & 108 & 59 & 141 & 21 & 33 & 11 & 58 & 12 \\
5 & 101 & 60 & 200 & 30 & 53 & 33 & 99 & 14 & 97 & 11 & 149 & 25 & 52 & 4 & 84 & 17 \\
\hline
\end{tabular}

Table 1. Number of correct and error trials (mean, minimum, maximum and SD) for each task in Experiments 1-5. Task 1 is the task associated with higher confidence (boost selected / correct box for Experiments 1-4 and boost incorrect box for Experiment 5). Task 2 is the task associated with lower confidence (boost alternative / incorrect box for Experiments 1-4 and boost correct for Experiment 5).

\begin{tabular}{|c|c|c|c|c|c|c|c|c|c|c|c|c|c|c|c|c|}
\hline \multirow[b]{3}{*}{ Exp. } & \multicolumn{8}{|c|}{ Reported Error } & \multicolumn{8}{|c|}{ Reported Correct } \\
\hline & \multicolumn{4}{|c|}{ RI1 } & \multicolumn{4}{|c|}{ RI2 } & \multicolumn{4}{|c|}{$\mathrm{RC} 1$} & \multicolumn{4}{|c|}{$\mathrm{RC} 2$} \\
\hline & $M$ & Min & $\operatorname{Max}$ & $S D$ & $M$ & Min & $\operatorname{Max}$ & $S D$ & $M$ & Min & $\operatorname{Max}$ & $S D$ & $M$ & Min & Max & $S D$ \\
\hline 1 & 26 & 9 & 76 & 16 & 31 & 9 & 87 & 18 & 107 & 52 & 134 & 16 & 129 & 60 & 180 & 25 \\
\hline 2 & 29 & 15 & 55 & 10 & 33 & 19 & 56 & 10 & 109 & 84 & 129 & 12 & 124 & 90 & 144 & 16 \\
\hline 3 & 27 & 9 & 57 & 13 & 30 & 10 & 64 & 14 & 112 & 75 & 139 & 16 & 131 & 110 & 150 & 12 \\
\hline 4 & 34 & 20 & 54 & 9 & 38 & 20 & 54 & 9 & 111 & 96 & 132 & 10 & 124 & 104 & 144 & 10 \\
\hline 5 & 33 & 5 & 80 & 18 & 37 & 10 & 84 & 18 & 99 & 55 & 124 & 18 & 122 & 62 & 175 & 26 \\
\hline
\end{tabular}

Table 2. Number of trials according to reported accuracy $(\mathrm{RI}=$ reported incorrect, $\mathrm{RC}=$ reported correct) and median splits ( 1 = below median confidence, 2 = above median confidence) within each accuracy group for Experiments 1-5. Note that for Experiment 4 trial numbers are based on objective, rather than reported accuracy. Data from participants that were excluded from analyses using this trial grouping are similarly excluded from the table. 
Task selection. Given that we successfully manipulated confidence across tasks, of critical interest was whether this confidence difference would be reflected in participants' task preferences. Consistent with this prediction, and as shown in Figure 2B, participants exhibited consistent preference for the boost selected task over the boost alternative one (mean proportion of boost selected choices $=0.54$, range $=0.47-0.76, S D=0.08$ ). 16 of 20 participants $(80 \%)$ chose the boost selected task more often than the boost alternative task, with this choice rate significantly different from chance level $\left(t(19)=2.37, p=.028, d_{z}=.53\right)$. The timecourse of the development of this preference (Figure 2C) suggested that it was established early in the block.

Error monitoring and confidence impact on task selection. To explore further this preference towards tasks associated with higher confidence, we examined how preferences emerge on a trial-by-trial basis as a function of participants' error monitoring and experienced confidence levels. Thus, we evaluated whether people tend to repeat a task more often if they believe they were correct (rather than incorrect), and separately whether they tend to repeat the same task if they experience higher confidence (vs. lower confidence) in correct decisions.

To test the effects of trial-by-trial error monitoring and confidence on task choice, trials were first divided according to reported accuracy, and then median confidence ratings were calculated separately for reported correct (RC) and reported incorrect (RI) trials, with trials in which participants reported guessing $(M=0.04, S D=0.03$ of trials $)$ excluded from this analysis. Median values were used to divide RC and RI trials into subsets with higher vs. lower than median confidence ratings (RI1, RI2, RC1, RC2; trial counts are listed in Table 2). For each subset, we calculated the likelihood that participants would repeat the same task (vs. switch) on the next trial-i.e., as a function of reported accuracy, and as a function of confidence level (see Figure 2D). Because we define confidence as subjective p(correct), we define a trial with a "sure incorrect" confidence rating as having the lowest possible decision 
confidence (even though the participant is expressing certainty in this evaluation). A $2 \times 2$ repeated measures ANOVA was performed on task repetition probability, with reported accuracy (correct, incorrect) and confidence rating (below median, above median) as factors. Significant effects were found for reported accuracy $\left(F(1,19)=7.30, p=.014, \eta_{p}^{2}=.28\right)$, with a higher proportion of task repetitions following trials that were reported as correct than incorrect, and for the interaction between reported accuracy and confidence $(F(1,19)=5.62, p$ $\left.=.029, \eta_{p}^{2}=.23\right)$. There was no significant main effect of confidence $(F<1)$. Follow-up analysis revealed that for trials reported as correct, participants were significantly more likely to repeat the same task after experiencing higher confidence in a decision $(t(19)=3.57, p=$ $\left..002, d_{\mathrm{z}}=0.80\right)$. There was no significant effect of confidence when trials were reported as incorrect $\left(t(19)=-1.14, p=.27, d_{\mathrm{z}}=-0.26\right)$, but interestingly the numerical trend was towards participants being more likely to repeat tasks, not less, when they experienced lower confidence (i.e. were more certain they had made an error).

Evidence strength and confidence impact on task selection. Our final analysis considered an alternative explanation of the above findings, which is that participants are showing a preference for situations associated with stronger evidence, rather than higher confidence. Our results show that confidence is increased when people receive post-decisional evidence that provides stronger support for (or less contradiction of) their decision, and that people prefer tasks with this evidence boost. With this manipulation, when choosing the boost selected box task, participants were presented with strong evidence when they responded correctly and weak evidence when they responded incorrectly. The opposite was true for the boost alternative box task. Because participants responded correctly more often than incorrectly (mean accuracy $=0.73, S D=0.08$ ), this means that the boost selected box task was also associated with stronger evidence overall than the boost alternative box task (mean dot difference of 28.50 vs. $20.03 ; S D=1.72$ vs. 1.65 , respectively, $t(19)=12.07, p<.001)$. 
To provide evidence that the emerging preference was not only the result of a bias towards stronger evidence, we fitted logistic regression models predicting task repetition on trial $\mathrm{N}+1$ as a function of evidence strength and confidence on trial $\mathrm{N}$, for reported correct and error trials separately, separately for each participant. Trials were divided by reported accuracy to allow the detection of differential effects of confidence, expected based on the interaction effect reported above. Evidence strength was calculated as the mean difference in dot numbers between the two boxes across frames in each trial, taking into account both pre- and postdecisional evidence. Note that although the mean dot difference between boxes was fixed across conditions, evidence strength varied across trials because of random variation in the number of dots presented in each stimulus frame around the overall mean values. Standard scores for evidence strength and confidence were computed for correct and incorrect trials separately before running the regression.

For correct trials, we observed positive slopes for confidence in 17 of 20 participants, indicating that participants were more likely to repeat task choice following decisions made with higher confidence, with the mean beta being significantly different from zero across participants $\left(M=0.40, S D=0.46, t(19)=3.94, p<.001, d_{z}=0.88\right)$. For incorrect trials we found the reverse relationship, with participants more likely to repeat tasks when they were less confident (i.e. more sure they had made a mistake). Slopes were negative for 16 participants and the mean beta significantly different from zero $(M=-0.21, S D=0.41, t(19)$ $=-2.34, p=0.03, d_{\mathrm{z}}=-0.52$ ). Evidence strength did not significantly predict repetitions in task choice: slopes were positive for 10 of 20 participants for both reported correct and error trials, and betas were not significantly different from zero across participants $(M=0.02, S D=$ $0.20 ; M=-0.14, S D=0.64$, for correct and error trials respectively; $t \mathrm{~s}<1$ ). These results support our interpretation that participants' bias for the boost selected task is a result of confidence preferences, above and beyond strong evidence. The results are also consistent with 
the error monitoring analysis presented above, demonstrating that confidence reliably predicts task repetitions for correct trials (or those perceived as such). For perceived incorrect trials, the effects of confidence on task choice are weaker, but the results are consistent with the numerical trend reported above.

\section{Discussion}

The results of Experiment 1 support our main hypothesis, with people choosing situations in which their confidence is higher more often. The effect overall was small $(M=$ .54 vs. .46 for the alternative task that was associated with lower confidence) but was reliable, apparent in a large majority of participants, and was apparent even though the impact of the evidence manipulation on confidence itself was very subtle. Furthermore, at the trial level, task selection on trial $\mathrm{N}+1$ was reliably predicted by confidence on trial $\mathrm{N}$, with participants more likely to repeat their task choice after a perceived correct decision made with high vs. low confidence. Interestingly, for trials perceived as incorrect, participants were more likely to repeat their choice when they were more certain they had made a mistake (i.e. lower in confidence). However, these results should be interpreted with care as they were less robust, were not significant in the ANOVA, and are based on the relatively small number of trials that were perceived as errors. It is important to note that, consistent with previous studies showing that people find errors aversive (Hajcak \& Foti, 2008), participants were also overall less likely to repeat their task choice following trials they judged as incorrect. Thus, if participants reported more errors in one task compared to another, this would lead to a lower proportion of trials spent on this task. We suspected that the demonstrated preference might be driven in part by these differences in error detection. Indeed, participants judged a significantly larger proportion of trials as being errors in the boost alternative compared to the boost selected box task $(M=0.23$ vs. $0.15, S D$ s $=0.11$ for the boost alternative and boost selected tasks 
respectively, $\left.t(19)=5.44, p<.001, d_{z}=1.22\right)$. Experiments 2 and 3 therefore assessed whether the reported effect would persist in situations in which error monitoring differences were less pronounced.

\section{Experiments 2 and 3}

Experiments 2 and 3 were designed to minimize differences in error monitoring across the tasks being selected, to allow us to investigate specifically the influence of confidence on task preferences. Thus, in Experiment 2, post-decisional evidence was manipulated only following correct responses, boosting evidence in favor of the correct response (i.e. in favor of participants' decisions) for one cue and boosting evidence in favor of the incorrect response (i.e. against participants' decisions) for the other. In Experiment 3, post-decisional evidence was manipulated regardless of participants' accuracy on the trial, so that post-decisional evidence was boosted in favor of the objectively correct response for one cue, and in favor of the incorrect response for the other. Since the majority of trials were correct, we expected that boosting the evidence in favor of the correct responses would still result in higher confidence overall, but also that detecting errors would be easier in this task, allowing us to test if the preference persists even under these conditions.

\section{Methods}

Participants. 20 participants took part in Experiment 2 (12 females, ages 18-28 years, mean age $=22.8, \mathrm{SD}=2.77) .21$ participants took part in Experiment 3, with one participant removed from the final sample due to non-compliance with instructions (always selecting the upper cue), resulting in a final sample of 20 participants (12 females, ages 18-33 years, mean age $=23.6, S D=4.07)$. All participants had normal or corrected-to-normal vision. They 
provided written informed consent and were compensated with course credit or payment for their participation.

Stimuli and Procedure. The overall design of Experiments 2 and 3 was identical to Experiment 1, only differing in the nature of the post-decisional evidence manipulation. Thus, on all trials in both experiments, the mean strength of pre-decisional evidence was fixed at the same level as in Experiment $1\left(M_{C}=212, M_{I}=188\right)$. In Experiment 2, evidence was manipulated only after correct responses. One cue was associated with a high-confidence task, in which post-decisional evidence was boosted after correct responses so that the difference between dot numbers grew $\left(M_{C}=222, M_{I}=178\right)$ - the boost correct box task. The second cue was associated with a low-confidence task, in which post-decisional evidence was reduced following correct responses, so that the difference between the boxes decreased $\left(M_{C}=202\right.$, $M_{I}=198$ ) - the boost incorrect box task. Following errors, the evidence remained the same as in the pre-decision stage in both tasks $\left(M_{C}=212, M_{I}=188\right)$, so as to avoid making error detection easier in one task compared to the other. In Experiment 3, post-decisional evidence was manipulated similarly, but regardless of participants' accuracy on the trial. Specifically, one cue was associated with a task in which post-decisional evidence was always boosted in favor of the objectively correct response (the boost correct box task, $M_{C}=222, M_{I}=178$ ), and the second cue was associated with a task in which evidence strength was always reduced (boost incorrect box task, $M_{C}=202, M_{I}=198$ ), following both correct and incorrect trials (note that following correct trials, evidence manipulation in Experiments 2 and 3 was identical). With this manipulation, we expected that participants would experience higher confidence in their correct responses but lower confidence in their errors (i.e., stronger error detection) in the boost correct compared to the boost incorrect task. As in Experiment 1, for all conditions the correct square had on average more dots than the incorrect square in the postdecisional (as well as pre-decisional) period. 


\section{Results}

Manipulation effects on error monitoring. Our first analyses assessed whether the manipulations in Experiments 2 and 3 were effective in minimizing differences in error monitoring between the tasks. To this end, the difference in the proportion of reported errors between the tasks was calculated for each participant. In Experiment 2, a numerically small but significant difference still remained (mean proportion of reported errors in boost correct task $=$ .19 , in boost incorrect task $=.22, t(19)=2.30, p=.03, d_{\mathrm{z}}=.52$ ). A between participant t-test was performed to compare the size of these differences between Experiment 1 and $2(M=$ $0.073, S D=0.06, M=0.025, S D=0.05$ for Experiments 1 and 2 respectively). As expected, the difference was smaller in Experiment $2(t(38)=2.75, p<.01, d=0.86)$. This indicates that, though a small difference remained, the manipulation was successful in reducing the differences in error monitoring between the tasks. In Experiment 3, no significant difference was found between the proportion of errors reported in the high- and low-confidence tasks ( $M$ $=.19$ for both tasks, $S D=.08$ and .10 in the boost correct and boost incorrect tasks respectively; $t<1)$.

Manipulation effects on performance and confidence. As expected, no significant differences were found in performance between the tasks. Participants responded on time to a similar proportion of trials (Experiment 2: $M=0.95$ vs $0.96, S D=0.05$ and $0.04, t(19)=-1.68$, $p=0.11, d_{\mathrm{z}}=-0.38$; Experiment 3: $M=0.96$ vs 0.97, $S D s=0.02, t(19)=-1.11, p=0.28, d_{\mathrm{z}}=$ -0.25 , for the boost correct and boost incorrect tasks respectively). For trials with on-time responses, no reliable differences were found in accuracy levels between the tasks (Experiment 2: $M=0.72$ vs $0.73, S D=0.07$ and $0.08, t<1$; Experiment $3: M=0.75$ vs $0.72, S D=0.08$ and 0.07, $t(19)=1.68, p=0.11, d_{z}=0.38$, for the boost correct and boost incorrect tasks respectively). Similarly, no reliable differences were found in mean reaction times (Experiment 
2: $M=586$ vs $585 \mathrm{~ms}, S D=56$ and $61 \mathrm{~ms}$; Experiment $3: M=600$ vs. $604 \mathrm{~ms}, S D=32$ and $24 \mathrm{~ms} ; t s<1)$.

Figure 3A shows mean confidence ratings in Experiment 2, which varied across tasks as predicted. A $2 \times 2$ repeated measure ANOVA on these ratings revealed main effects for task $\left(F(1,19)=9.45, p=.006, \eta_{p}^{2}=.33\right)$ and for accuracy $\left(F(1,19)=154.34, p<.001, \eta_{p}^{2}=.89\right)$, and a reliable interaction between these factors $\left(F(1,19)=30.83, p<.001, \eta_{p}^{2}=.62\right)$. Pairwise comparisons revealed a significant difference in confidence between tasks for correct responses $\left(M=24.17\right.$ vs. $19.23, S D=8.12$ and $7.30, t(19)=5.63, p<.001, d_{z}=1.26$ for the boost correct and boost incorrect tasks, respectively), but not for incorrect responses ( $M=-7.87$ vs. -6.82 , $S D=8.41$ and $\left.7.94, t(19)=-1.35, p=.19, d_{\mathrm{z}}=-0.30\right)$, as expected given that we manipulated evidence only following correct trials in this experiment. A corresponding analysis of confidence ratings in Experiment 3 (Figure 4A) revealed no significant main effect of task $(F$ $\left.(1,19)=1.13, p=.30, \eta_{p}^{2}=.06\right)$, a significant main effect of accuracy $(F(1,19)=148.70, p<$ $\left..001, \eta_{p}^{2}=.89\right)$, and a reliable interaction between the two factors $(F(1,19)=93.92, p<.001$, $\left.\eta_{p}^{2}=.83\right)$. Follow-up analyses revealed that, as expected, confidence was significantly higher in the boost correct task than the boost incorrect task when participants responded correctly $(M$ $=28.24$ vs $21.12, S D=5.80$ and $\left.6.86, t(19)=6.15, p<.001, d_{z}=1.38\right)$, and this pattern reversed 

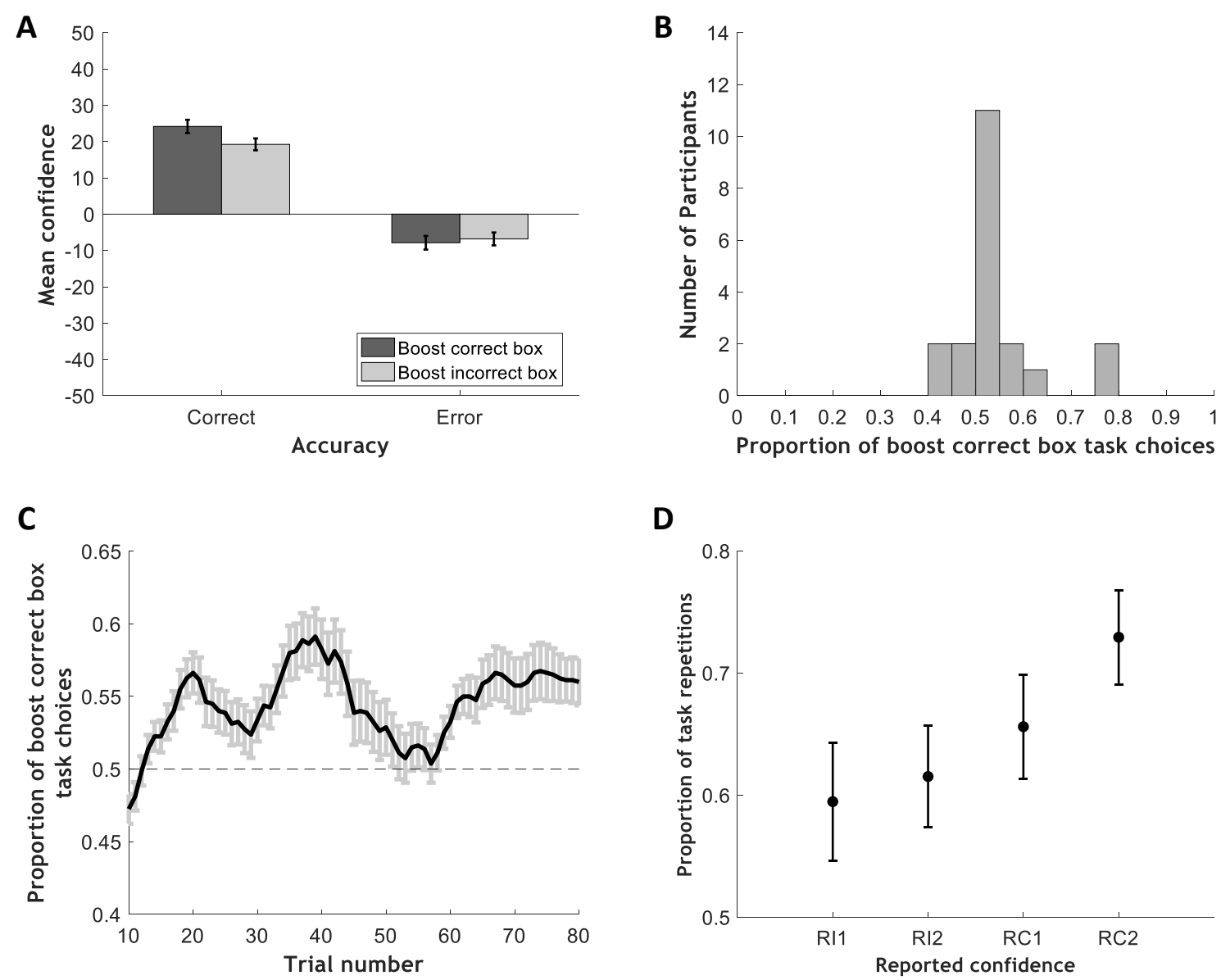

Figure 3. Results of Experiment 2. A, Mean confidence ratings for the boost correct box and boost incorrect box tasks, divided according to trial accuracy. Evidence was manipulated only following correct responses. B, Distribution of individual participants' proportion of boost correct box task choices $(n=20)$. $C$, Ten-trial running average showing the proportion of boost correct box task choices across blocks. Dashed line represents chance level. $\boldsymbol{D}$, Mean proportion of task repetitions according to reported confidence. Trials were first divided into groups according to reported accuracy $(\mathrm{RI}=$ reported incorrect, $\mathrm{RC}=$ reported correct), followed by a division according to median splits within the accuracy group ( 1 = below median confidence, 2 = above median confidence). Error bars represent SEMs.

on incorrect trials $\left(M=-12.26\right.$ vs $-3.10, S D=10.79$ and $11.48, t(19)=-6.63, p<.001, d_{z}=-$ 1.48). Because participants were correct on most trials (mean accuracy $=0.74, S D=0.07$ ), mean confidence across all trials was significantly higher in the boost correct task $(M=18.4$ vs. 14.6 in the boost incorrect task, $S D=5.45$ and 6.92 , respectively; $t(19)=2.85, p=.01, d_{z}$ $=0.64)$. Note that one participant who showed a strong preference towards the boost correct 
box task only had a small number of trials to include in these analyses $(8$ corrects and 2 errors, as listed in Table 1). However, there are no meaningful changes to the results when excluding this participant.
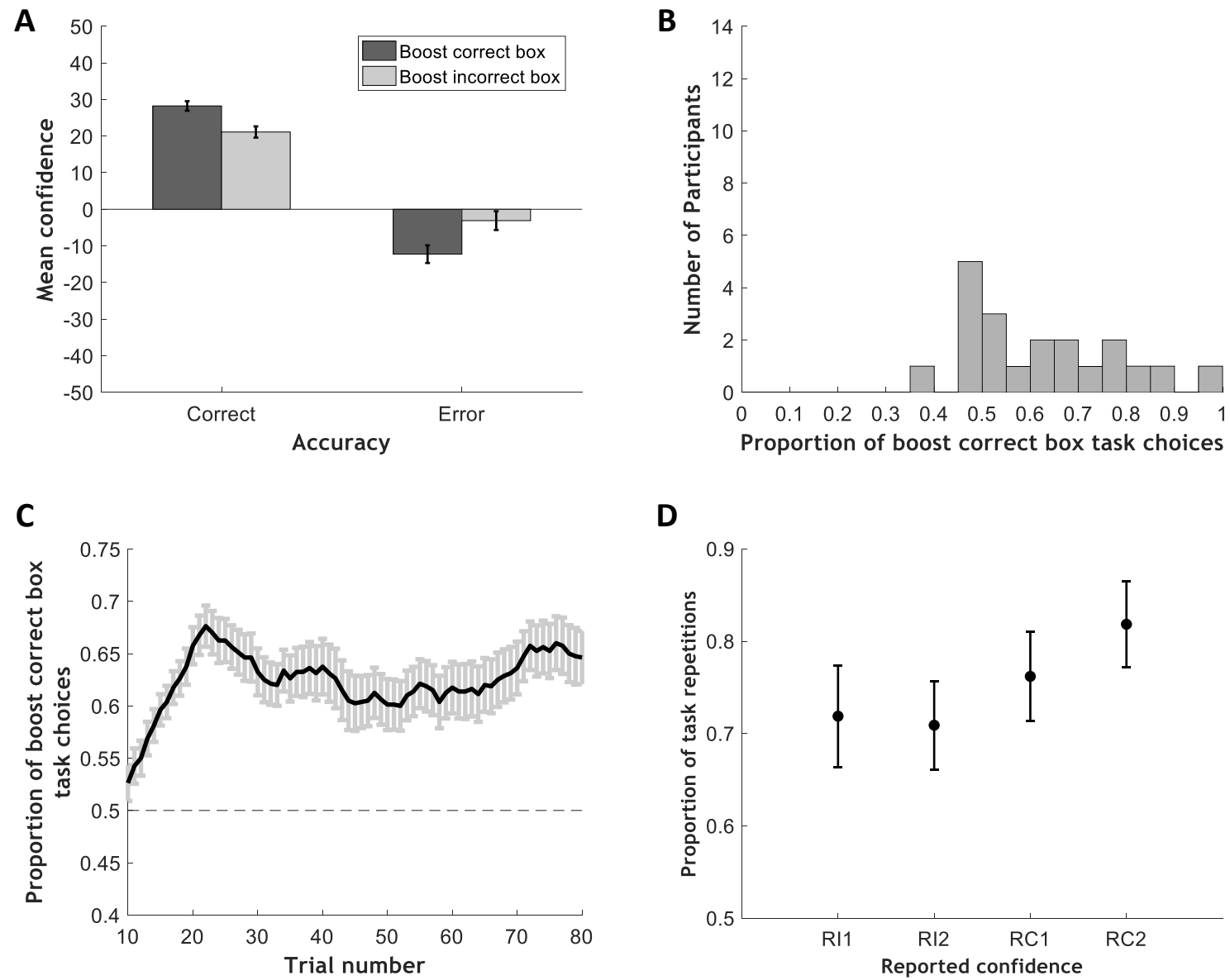

Figure 4. Results of Experiment 3. A, Mean confidence ratings for the boost correct box and boost incorrect box tasks, divided according to trial accuracy. $\boldsymbol{B}$, Distribution of individual participants' proportion of boost correct box task selections $(n=20)$. C, Ten-trial running average showing the proportion of boost correct box task selections across blocks. Dashed line represents chance level. $D$, Mean proportion of task repetitions according to reported confidence. Trials were first divided into groups according to reported accuracy ( $\mathrm{RI}$ = reported incorrect, $\mathrm{RC}=$ reported correct), followed by a division according to median splits within the accuracy group $(1=$ below median confidence, 2 = above median confidence). Note: Error bars represent SEMs.

Task selection. Results of the critical analysis of task selections are shown in Figures 3 and 4 (panels B and C), which exhibit a preference for the task associated with higher average 
confidence in both experiments. In Experiment 2, 16 of 20 participants (80\%) chose the boost correct box task more often than the boost incorrect task (see Figure 3B, 3C). The mean proportion of boost correct choices was 0.54 (range $=0.41$ to $0.78, \mathrm{SD}=0.09$ ), a preference that was significantly different from chance level $\left(t(19)=2.11, p<.05, d_{\mathrm{z}}=0.47\right)$. In Experiment 3, 13 of 20 participants $(65 \%)$ selected the boost correct task more than the boost incorrect one. The mean proportion of boost correct selections was 0.62 across participants (range $=0.35$ to $0.97, \mathrm{SD}=0.16$ ), again significantly different from chance level $(t(19)=3.30$, $p<.01, d_{\mathrm{z}}=0.74$; see Figure 4B, 4C). The preference for the task associated with higher average confidence was less consistent across participants in Experiment 3 than Experiment 2, but of larger numerical magnitude (and more consistent across trials of the block) in Experiment 3 (compare Figures 3C and 4C), with some participants showing an overwhelming preference for the boost correct task over the boost incorrect task.

Error monitoring and confidence impact on task selection. We performed an identical analysis to that described above for Experiment 1 to assess the trial-by-trial influence of subjective confidence on task choice: Trials were divided according to reported accuracy and then relative to each participant's median confidence rating within each of these trial subsets (RI1, RI2, RC1, RC2; guess trials were excluded; see Table 2 for trial counts), and then we calculated for each trial subset the likelihood that participants would repeat task choice (vs. switch) on the subsequent trial. We subjected these data to a $2 \times 2$ repeated measures ANOVA on repetition probability with reported accuracy (correct, incorrect) and confidence rating (below median, above median) as factors, separately for each experiment. In Experiment 2, significant effects were found for reported accuracy $\left(F(1,19)=21.27, p<.001, \eta_{p}^{2}=.53\right)$ and for confidence $\left(F(1,19)=10.31, p=.005, \eta_{p}^{2}=.35\right)$, with participants repeating their task choice more often following correct trials, and when their confidence was high. The interaction between reported accuracy and confidence was not significant $\left(F(1,19)=2.96, p=.10,, \eta_{p}^{2}=\right.$ 
.13), but the numerical trend was for a larger impact of correct-trial confidence on task repetition. Because it was of interest whether effects of confidence are observed for correct and incorrect trials separately, we ran pairwise comparisons that revealed a significant difference in repetition likelihood following trials with high- vs. low-confidence on reported-correct trials $\left(t(19)=5.38, p<.001, d_{z}=1.20\right)$, with participants repeating tasks more often following decisions made with high confidence. As in Experiment 1, no significant effect of confidence was found on reported incorrect trials (see figure $3 \mathrm{D}, t<1$ ), and the small numerical trend was in the opposite direction to that observed in the previous experiment, so that participants repeated more after higher confidence decisions.

In Experiment 3, one participant whose data could not be binned according to median confidence was excluded from the analysis (for this participant median confidence rating for incorrect trials $=-50$, leading to no trials in the incorrect below-median bin). Task repetition likelihood varied reliably as a function of reported accuracy $\left(F(1,18)=9.98, p=.005, \eta_{p}^{2}=\right.$ $.36)$, but not confidence $\left(F(1,18)=2.09, p=.17, \eta_{p}^{2}=.10\right)$, or the interaction between accuracy and confidence $\left(F(1,18)=3.75, p=.07, \eta_{p}^{2}=.17\right)$. Because we expected similar effects to those found in previous experiments, analyses were run to test for separate effects of confidence on correct and error trials. Indeed, here too we found a significant difference in repetition proportion following high- vs. low-confidence decisions on reported correct trials $(t(18)=3.83$, $p=.001, d_{z}=.88$ ), with participants repeating their task choice more often after high confidence judgments. No such effect was found for reported incorrect trials $(t<1$, Figure 4D) and, as in Experiment 1, the numerical trend was in the opposite direction.

Evidence strength and confidence impact on task selection. Similar to Experiment 1, our manipulation in the current experiments resulted in stronger evidence (greater average difference in number of dots between the two boxes) in tasks associated with higher average confidence (Experiment $2: M=30.80$ vs. $17.26 ; S D=0.82$ and 0.90 , for the boost correct vs. 
boost incorrect tasks; $t(19)=39.13, p<.001$; Experiment $3: M=33.15$ vs. $14.60 ; S D=0.52$ and 0.57 , for the boost correct vs. boost incorrect tasks, $t(19)=96.73, p<.001)$. To test for the unique contribution of confidence while controlling for these differences in evidence strength, we ran logistic regression models using evidence strength and confidence on trial $\mathrm{N}$ as predictors of participants' task selection repetitions on trial $\mathrm{N}+1$, separately for each participant and for reported correct and error trials, for the data from Experiments 2 and 3.

In Experiment 2, for perceived correct trials, 19 of 20 participants had positive slopes for confidence, indicating they were more likely to repeat task choice following decisions made with higher confidence, with the mean beta being significantly different from zero across participants $\left(M=.30, S D=0.17, t(19)=7.66, p<.001, d_{\mathrm{z}}=1.71\right)$. For evidence, slopes were positive in 13 of 20 participants, and numerically participants tended to be more likely to repeat their task preference following correct-response trials with stronger evidence, but the mean slope was not significantly different from zero across participants $(M=.12, S D=.28, t(19)=$ $1.92, p=.07, d_{\mathrm{z}}=0.43$ ). For error trials, neither confidence (13 positive slopes, $M=.07, S D$ $=.34)$ nor evidence strength (14 positive slopes, $M=.05, S D=.54)$ reliably predicted repeating task choice on the subsequent trials $(t \mathrm{~s}<1)$.

In Experiment 3, we once again found that higher correct-trial confidence was associated with increased likelihood of repeating task choice on the subsequent trial, with 18 of 20 participants having a positive beta value in the logistic regression, the mean value of which was reliably larger than zero $\left(M=0.49, S D=0.85, t(19)=2.54, p=.02, d_{z}=0.57\right)$. As in Experiment 2, participants tended to be more likely numerically to repeat their task preference following correct-response trials with stronger evidence, but this effect was not reliable (positive betas in 14 of 20 participants, $M=.19, S D=.49, t(19)=1.71, p=.10, d_{\mathrm{z}}=$ 0.38 ). For error trials, two participants whose data was not suitable for a logistic regression were removed from the analysis. Neither confidence (positive betas for 10 participants, $M=$ 
$0.01, \mathrm{SD}=0.52, t<1$ ) nor evidence strength (positive betas for 10 participants, $M=0.23, S D$ $\left.=0.59, t(17)=1.70, p=.11, d_{z}=0.40\right)$ reliably predicted repeating task choice on the subsequent trials. Thus, in line with the results from Experiment 1, Experiments 2 and 3 confirm that confidence influenced participants' task choices in a trial-to-trial manner, above and beyond the effects of trial-varying evidence strength.

\section{Discussion}

Our aim in Experiments 2 and 3 was to replicate the key findings from Experiment 1, but do so using conditions in which the effects of error monitoring should be minimized. We varied the post-decisional evidence manipulation so that errors would not be easier to detect in the task associated with lower compared to higher confidence: In Experiment 2, boosting evidence in favor of the correct box only after correct responses reduced the difference in error detection rates across conditions. In Experiment 3, boosting evidence in favor of the objectively-correct option regardless of response accuracy led to comparable rates of error detection across conditions. Even under these conditions, participants still exhibited a preference towards selecting the cue associated with higher average decision confidence, lending further support to our main hypothesis. Furthermore, our regression analyses replicated the finding from Experiment 1 that participants' trial-by-trial confidence ratings predict their subsequent task choices even when controlling for the strength of evidence presented.

An interesting question regarding the demonstrated preference is the degree to which it depends on the presence (or lack) of external feedback in Experiments 1-3. If people use confidence solely as an internal monitoring system, it might be predicted that the effects of confidence judgments would be redundant when external feedback is present. In this case, the effects previously demonstrated here might be expected to disappear. If, however, confidence 
acts as a cost or benefit factor intrinsic to a decision, we might still expect to see its effects even in the presence of feedback. We addressed this question in Experiment 4.

\section{Experiment 4}

To evaluate the influence of feedback on the preference for tasks associated with higher average confidence, visual feedback of participants' first-order performance ("correct", "incorrect", "too slow") was provided at the end of each trial in this experiment. Evidence in this experiment was manipulated in the same way as described above for Experiment 3, with a post-decision evidence boost in favor of either the objectively correct or incorrect response regardless of the accuracy of the participant's decision on the trial. We expected that participants would develop a preference for the high-confidence task (boost correct condition) even when feedback is present. This prediction was based on the results described above, indicating that people are not only biased against tasks in which they make errors, but also in favor of tasks in which they experience higher confidence in correct responses, an effect that might persist even if participants are given objective information about whether their response was correct or not.

\section{Methods}

Participants. 20 participants took part in Experiment 4 (14 females, ages 18-26 years, mean age $=19.9, S D=2.28$ ). All participants had normal or corrected to normal vision. They provided written informed consent, and were compensated with course credit or payment for their participation.

Stimuli and Procedure. The design was similar to that of Experiment 3, except that feedback was given at the end of each trial. Thus, pre-decisional evidence was once again fixed across all trials $\left(M_{C}=212, M_{I}=188\right)$, while post-decisional evidence was boosted in favor of the objectively correct response for one cue (boost correct box task, $M_{C}=222, M_{I}=178$ ) 
and for the objectively incorrect response for the other (boost incorrect box task, $M_{C}=202$, $\left.M_{I}=198\right)$. Immediately after participants registered their confidence rating on each trial, or after the $800 \mathrm{~ms}$ time limit if no response was made, on-screen feedback indicated whether the participant's response was "correct", "incorrect", or "too slow" for $400 \mathrm{~ms}$, before the onset of the next trial.

\section{Results}

Manipulation effects on performance and confidence. Proportions of on-time responses for the two tasks were again well-matched $(M=.96 S D=.02$ vs. $M=.96, S D=.03$ for boost correct and boost incorrect tasks respectively, $t<1$ ), as were accuracy levels and response times for trials with on-time responses (accuracy levels: $M=0.77, S D=0.06$, for both tasks, $t<1$; response times: $M=593$ vs. $588 \mathrm{~ms}, S D=39$ and $41, t(19)=1.53, p=0.14, d_{\mathrm{z}}=$ 0.34). A 2 × 2 repeated measures ANOVA on confidence ratings (Figure 5A and Table 1) revealed main effects for task $\left(F(1,19)=4.67, p=.04, \eta_{p}^{2}=.20\right)$ and accuracy $(F(1,19)=$ $\left.179.65, p<.001, \eta_{p}^{2}=.90\right)$, and a reliable interaction between these factors $(F(1,19)=38.46$, $p<.001, \eta_{p}^{2}=.67$ ). Pairwise comparisons revealed that, as expected, confidence was significantly higher in the boost correct compared to boost incorrect task for correct trials $(t(19)$ 
$\left.=7.71, p<.001, d_{\mathrm{z}}=1.72\right)$, but following incorrect responses this pattern was reversed $(t(19)$

$=-4.75, p<.001, d_{\mathrm{z}}=-1.06$ ). Participants were correct on most trials (mean accuracy $=0.77$,

$\mathrm{SD}=0.06$ ), leading to significantly higher confidence overall in the boost correct compared to boost incorrect task $\left(\mathrm{M}=20.01\right.$ vs. $17.92, \mathrm{SD}=6.01$ vs. $\left.6.12 ; t(19)=3.12, p<.01, d_{\mathrm{z}}=0.67\right)$.

A

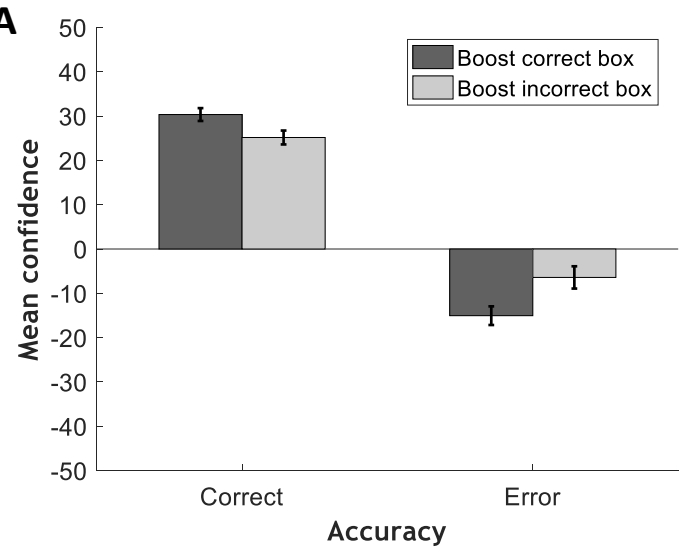

C

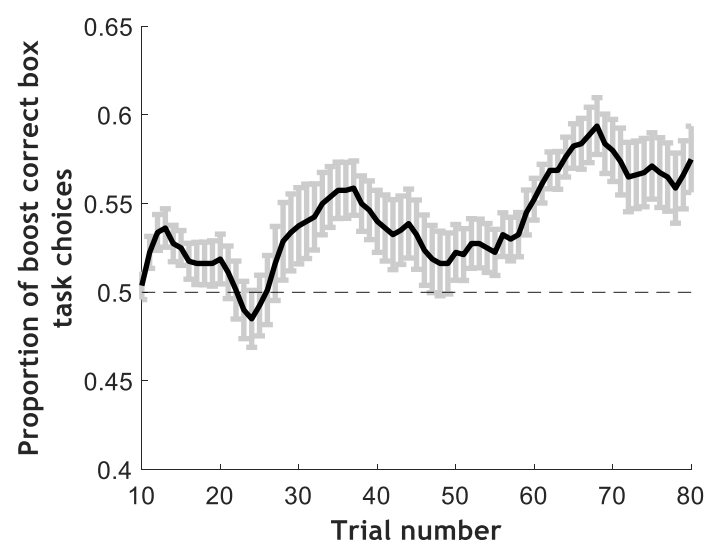

B

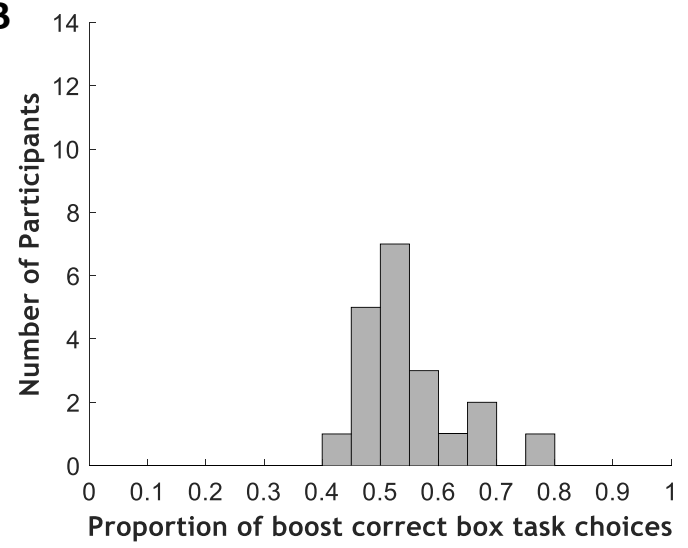

D

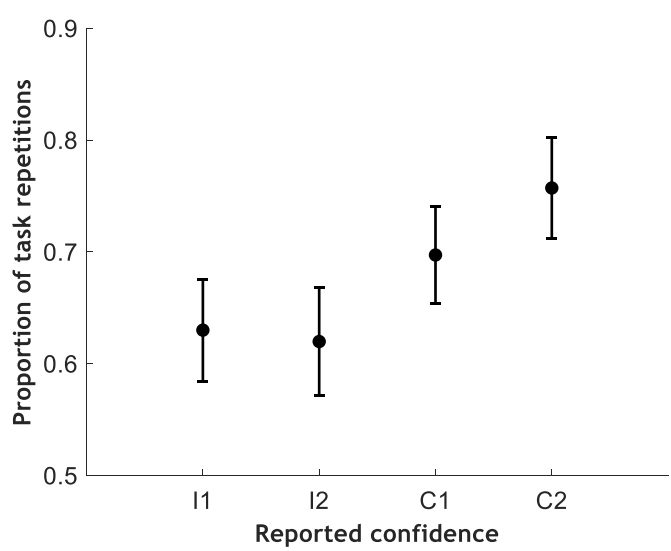

Figure 5. Results of Experiment 4. A, Mean confidence ratings for the boost correct box and boost incorrect box tasks, divided according to trial accuracy. $\boldsymbol{B}$, Distribution of individual participants' proportion of boost correct box task selections $(n=20)$. $C$, Ten-trial running average showing the proportion of boost correct box task selections across blocks. Dashed line represents chance level. $\boldsymbol{D}$, Mean proportion of task repetitions according to reported confidence. Trials were first divided into groups according to objective accuracy ( $I=$ incorrect, $C=$ correct), followed by a division according to median splits within the accuracy group ( 1 = below median confidence, 2 = above median confidence). Error bars represent SEMs. 
Task selection. As shown in Figure 5, participants' preference for tasks in which they experience higher confidence persisted even when they received objective feedback on their performance on each trial. The preference was perhaps somewhat weaker than in the previous experiments, being evident in 12 out of the 20 participants $(60 \%$, Figure $5 \mathrm{~B})$ and being evident consistently only towards the end of each block (Figure 5C). On average, participants chose the boost correct task more often than boost incorrect task (mean proportion $=0.54$, range $=$ $0.41-0.77, S D=0.08$ ) at a rate that was significantly above chance level across participants $\left(t(19)=2.18, p=.04, d_{\mathrm{z}}=0.49\right)$.

Error monitoring and confidence impact on task selection. We performed a similar analysis to that described above for Experiments 1-3. However, since participants received feedback on their accuracy, trials were divided according to objective rather than reported accuracy. Importantly, this allowed us to assess the degree to which subjective confidence within a known accuracy level predicted task choice on the subsequent trial. The median confidence rating for correct and incorrect trials was computed, and trials divided relative to this rating (I1, I2, C1, C2, see Table 2 for trial counts). Two participants whose data could not be organized in this way were excluded from the analysis (median confidence rating for incorrect trials $=-50$ ). A $2 \times 2$ repeated measures ANOVA was then performed on repetition probability, with objective accuracy (correct, incorrect) and confidence rating (below median, above median) as factors (see Figure 5D). Significant effects were found for accuracy $(F(1,17)$ $\left.=16.73, p<.001, \eta_{p}^{2}=.50\right)$, and for the interaction between accuracy and confidence $(F(1,17)$ $\left.=6.24, p=.023, \eta_{p}^{2}=.27\right)$, but not for confidence $\left(F(1,17)=4.34, p=.053, \eta_{p}^{2}=.20\right)$. Followup pairwise comparisons revealed a significant difference in repetition probability following high- vs. low-confidence decisions on correct trials $\left(t(17)=4.56, p<.001, d_{z}=1.11\right)$, with participants repeating tasks more often after high confidence judgments. No significant effect 
was found for incorrect trials $(t<1)$, with repetition probability very similar regardless of participants' rated confidence on the previous trial.

Evidence strength and confidence impact on task selection. The evidence manipulation in Experiment 4 was identical to that used in Experiment 3, and resulted in similar evidence strengths, with stronger evidence in the task associated with higher confidence $(M=$ 33.34 vs. $14.79 ; S D=0.50$ and 0.70 for the boost correct and boost incorrect tasks, respectively, $t(19)=79.03, p<.001)$. Logistic regression models predicting repetitions in task choice on trial $\mathrm{N}+1$ based on the evidence strength and reported confidence on trial $\mathrm{N}$ were run for each participant, for objectively correct and error trials separately. 14 of 20 participants had positive slopes for confidence on correct trials, and the beta values were significantly different from zero, indicating that higher confidence was predictive of an increased likelihood of repeating task choice on the subsequent trial $\left(M=0.24, S D=0.30, t(19)=3.58, p=.002, d_{\mathrm{z}}=0.80\right)$, even when objective feedback was interposed. Only 10 participants had positive slopes for confidence following error trials, and this did not predict switching $(M=-0.01, S D=0.37, t<$ 1). No significant effects were found for evidence strength predicting subsequent task choice (correct trials: 14 participants had positive slopes, $t(19)=1.55, p=.14, d_{z}=0.35$; incorrect trials: 14 participants had positive slopes, $t(19)=1.16, p=.26, d_{\mathrm{z}}=0.26$ ).

\section{Discussion}

This experiment evaluated whether a bias towards tasks associated with higher confidence is restricted to situations in which objective feedback is absent (and, hence, in which participants are inherently reliant on their own subjective evaluations), or whether this bias might persist even when participants are told on each trial whether their response is correct or incorrect. The results demonstrate that, even when feedback is present and accuracy levels are very closely matched, participants were biased toward selecting the task associated with higher 
average confidence more often than a task associated with lower confidence levels. Our results further show that even when participants know they are correct, they are more likely to choose the same task on the next trial following decisions in which they felt more confident. This result provides strong support for the role of confidence as an internal cost or benefit factor in decisions, acting as a fine-grained cue that guides task choices beyond binary error monitoring. Experiments 1-4 all provide evidence for a consistent effect of confidence on task repetitions, above and beyond evidence. However, in all four experiments the cue associated with higher confidence was also associated with stronger evidence. In the final experiment described below, we pitted these factors against one another to examine the influences of highconfidence and strong-evidence when associated with different tasks.

\section{Experiment 5}

To study the independent effects of high-confidence and strong-evidence on task choices, in Experiment 5 we manipulated post-decisional evidence only following incorrect responses (a complementary paradigm to the one used in Experiment 2, in which evidence was manipulated only following correct responses). Here, following incorrect responses, one cue was associated with a boost in evidence strength (i.e. larger dot difference in favor of the correct answer), which should make it easier to detect errors and thus reduce overall confidence. The

other cue was associated with a decrease in evidence strength, which should make it harder to detect errors and thus increase overall confidence. Therefore, evidence strength and confidence work in opposite directions in this experiment, with confidence manipulated only for incorrect responses, thus exploring the extremes of possible influences of confidence on task choice. The experiment followed the design of Experiments 1-3, in that participants did not receive feedback on each trial. 
Participants. 38 participants took part in Experiment 5 (25 females, ages 18-35 years, mean age $=24.29, S D=4.51)$. A larger sample was used because we expected the effect size of the task preference to be smaller in this study, given that we manipulated evidence strength on only a small subset of trials (those with incorrect responses) and pitted evidence strength and confidence effects against one another. Specifically, the chosen sample size provides power of 0.8 to detect an effect size of $d_{\mathrm{z}}=0.47$, which is the effect size observed in Experiment 2 - the experiment with the weakest effect out of the four reported above, and the one with a complementary manipulation of evidence strength varying only following a subset of trials. All participants had normal or corrected-to-normal vision, provided written informed consent, and were compensated with course credit or payment for their participation.

Stimuli and procedure. The experimental design was similar to that used in Experiments 1-3, differing in only two respects. First, post-decisional evidence was only manipulated following incorrect responses in the initial dot-judgment task. For one cue, evidence strength was reduced after incorrect answers, (boost incorrect box task, $M_{C}=$ 202, $M_{I}=198$ ), which should make it harder to detect errors and thus increase overall confidence. For the other cue, evidence was increased after incorrect trials, which should lead to lower confidence (boost correct box task, $M_{C}=222, M_{I}=178$ ). Following correct responses, evidence strength remained as in the pre-decision stage. The second procedural difference in this experiment was that we used a reduced evidence strength in the pre-decision stage compared to the previous experiments, with dot number distributions centered around $M_{C}=208, M_{I}=192$ (which was again fixed across all trials regardless of the cue selected). We made this change because we were particularly interested in error trials, and therefore used weaker evidence to decrease initial decision accuracy.

\section{Results}


Manipulation effects on performance and confidence. As expected, accuracy was somewhat lower than in previous experiments but was closely matched between the two tasks $(M \mathrm{~s}=.65$ for both tasks, $S D=.08$ and .07 for the boost incorrect vs. boost correct tasks, $t<1)$. Participants made on-time responses in a similar proportion of trials $(M=.95 \mathrm{vs} .94, S D=.05$ and $\left..07, t(37)=1.54, p=0.13 d_{z}=0.25\right)$, with response times on these trials similar for both tasks $(M=603$ vs. $604 \mathrm{~ms}, S D=56$ and $61 \mathrm{~ms}, t<1)$. A $2 \times 2$ repeated measures ANOVA on confidence ratings (Figure 6A, Table 1) revealed reliable main effects of task $(F(1,37)=35.61$, $\left.p<.001, \eta_{p}^{2}=.49\right)$ and accuracy $\left(F(1,37)=152.65, p<.001, \eta_{p}^{2}=.81\right)$, and a significant interaction between these factors $\left(F(1,37)=50.23, p<.001, \eta_{p}^{2}=.58\right)$. Follow-up pairwise contrasts revealed that confidence was significantly higher in the boost incorrect than in the boost correct box task for incorrect trials $\left(t(37)=6.89, p<.001, d_{z}=1.12\right)$. As expected, no reliable confidence difference was found between tasks on correct trials, for which postdecisional evidence was not manipulated $\left(t(37)=1.55, p=0.13, d_{z}=0.25\right)$. When all trials were pooled together, participants expressed significantly higher confidence overall in the 

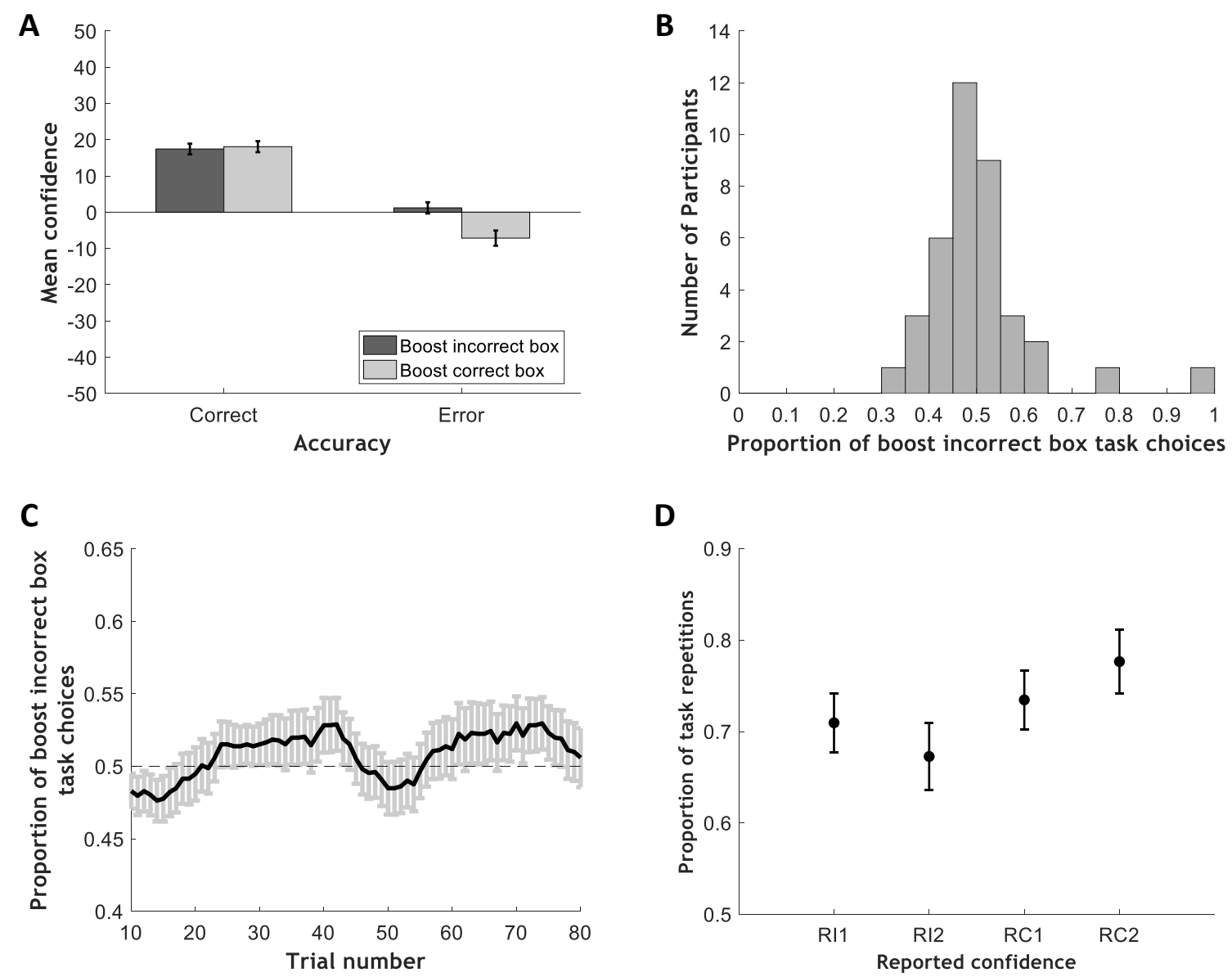

Figure 6. Results of Experiment 5. A, Mean confidence ratings for the boost incorrect box and boost correct box tasks, divided according to trial accuracy. B, Distribution of individual participants' proportion of boost incorrect box task selections $(n=38)$. $\boldsymbol{C}$, Tentrial running average showing the proportion of boost incorrect box task selections across blocks. Dashed line represents chance level. $D$, Mean proportion of task repetitions according to reported confidence. Trials were first divided into groups according to reported accuracy $(\mathrm{RI}=$ reported incorrect, $\mathrm{RC}=$ reported correct), followed by a division according to median splits within the accuracy group ( 1 = below median confidence, 2=above median confidence). Error bars represent SEMs.

boost incorrect task (weak evidence) compared to boost correct task (strong evidence; $M=$ 11.76 vs. $9.41, S D=8.14$ vs. 8.95 respectively; $\left.t(37)=3.64, p<.001, d_{\mathrm{z}}=0.59\right)$.

Task selection. With evidence strength and error-trial confidence acting in opposition across tasks, in this experiment we observed no consistent task preferences: 16 out of 38 participants selected the boost incorrect task, associated with higher confidence, more often $(42 \%$, Figure $6 B)$, with the overall average selection of this task not significantly different from 
chance level $(M=0.51, S D=0.11, t<1$, Figure $6 \mathrm{C})$. Thus, there was no evidence for the development of a consistent bias across participants in the current experiment.

Error monitoring and confidence impact on task selection. Although participants exhibited no consistent task preference, it remains possible that trial-varying confidence nevertheless guided participants' task choices. To assess this possibility, we again divided each participant's trials according to reported accuracy and relative to the median confidence ratings within each accuracy subset (RI1, RI2, RC1, RC2, guess trials excluded; see Table 2 for trial counts), then calculated the proportion of trials that participants repeated task choice after each trial type. A 2 x 2 repeated measures ANOVA on repetition probability with reported accuracy (correct, incorrect) and confidence ratings (below median, above median) as factors revealed no reliable effect of confidence $(F<1)$, but a significant main effect of reported accuracy $\left(F(1,37)=16.38, p<.001, \eta_{p}^{2}=.31\right)$ and a reliable interaction the two factors $(F(1,37)=9.71$, $p=.004, \eta_{p}^{2}=.21$, Figure $\left.6 \mathrm{D}\right)$. Follow-up pairwise comparisons revealed a significant difference in repetition probability following high- vs. low-confidence decisions on reported correct trials $\left(t(37)=3.78, p<.001, d_{z}=0.84\right)$, with participants more likely to repeat after judging a response as correct with high confidence. For reported incorrect trials, no significant effect was found $\left(t(37)=-1.64, p=0.11, d_{z}=-0.37\right)$ but the numerical trend pointed in the opposite direction, with participants more likely to repeat their task choice following trials in which confidence was lower.

Evidence strength and confidence impact on task selection. The average evidence strength for the boost incorrect box task was $M=14.04(S D=0.7)$, and for the boost correct task $M=20.51(S D=1.05)$, with a reliable difference found between tasks $(t(37)=25.72, p<$ $.001)$. Logistic regression models predicting repetitions in task choice on trial $\mathrm{N}+1$ based on the evidence strength and reported confidence on trial $\mathrm{N}$ were run for each participant, for reported correct and error trials separately. On reported correct trials, both confidence and 
evidence strength predicted choices. 32 of 38 participants had positive slopes for confidence on correct trials, and the beta values were significantly different from zero $(M=0.31, S D=$ $\left.0.36, t(37)=5.39, p<.001, d_{\mathrm{z}}=0.87\right)$, indicating once again that higher confidence predicted an increased likelihood of repeating task choice on the subsequent trial. 24 participants had positive slopes for evidence on correct trials, and these values were significantly different from zero $\left(M=0.08, S D=0.21, t(37)=2.23, p=.03, d_{z}=0.36\right)$, such that participants tended to repeat their task choice following trials in which they were presented with stronger perceptual evidence (i.e., easier decisions). No significant effects were found for the regression analysis of error trials, although paralleling the numerical trend apparent in the ANOVA reported above, 23 participants had negative slopes for confidence on error trials, repeating choices more for lower confidence $\left(M=-0.15, S D=0.53, t(37)=-1.72, p=.09, d_{z}=-0.28 ; t<1\right.$ for evidence strength betas).

\section{Discussion}

Experiment 5 investigated whether participants' preferences would be dominated by the strength of perceptual evidence or their degree of confidence in a task when these factors were set in opposition. If the task biases demonstrated in the previous experiments were driven mainly by a preference for strong evidence rather than high confidence, we would expect to see a bias towards the strong evidence (boost correct box) task emerge under the current conditions as well. As we did not find any such preference consistently across participants, this does not seem to be the case. Though the manipulation we used in the current experiment did not lead to the development of a consistent preference for the high confidence (boost incorrect box) task either, once again we found support for the role of confidence in task selection: As in our previous experiments, participants were more likely to repeat a task choice on the next trial if they made a decision on the current trial with higher confidence, an effect that was once 
again restricted to trials with correct responses. For incorrect trials we did not find that confidence or evidence strength predicted repeating task choice. These results are in agreement with participants' overall lack of a preference for either task, since in the current experiment we manipulated evidence (and thus confidence) only following incorrect trials.

\section{Questionnaire measures in Experiments 1-5}

Need for Cognition. An interesting question regarding the demonstrated biases is whether differences in their strength between individuals can be explained by trait differences in personality or cognitive style. To evaluate if preferences in the tasks were related to individuals' tendency to engage and enjoy cognitively effortful tasks, participants completed the Need for Cognition scale (Petty, Cacioppo \& Kao, 1984). We hypothesized that participants who chose the high-confidence task more often might score lower on the need for cognition scale. To guarantee a sufficient sample size, we combined the data from Experiments 1-4 (Experiment 5 was excluded due to the different nature of the manipulation), and performed a regression analysis with NFC scores and Experiment as predictors of high-confidence task choices. However, the results did not provide support for this suggestion $(F<1)$.

Behavioral Strategies and Metacognitive Awareness. To gain further insights into participants' subjective experience of the task and explicit use of choice strategies, we administered a debriefing questionnaire adapted from Kool et al., (2010) at the end of each experiment (see Table 3). We did not have specific predictions about participants' responses or how these responses would relate to behavior on the task, but report some exploratory analyses and examples we found to be of interest. Response samples are taken from participants in Experiment 1, which are representative of the responses we received across all experiments. 


\begin{tabular}{ll} 
No & Question \\
\hline 1 & How did you choose between the circles? \\
\hline 2 & Did you develop a preference for specific circles? Y/N \\
\hline 3 & If you replied yes, why? What was the difference between the circles? \\
\hline 4 & \begin{tabular}{l} 
Did you find the task associated with some circles to be easier? Y/N \\
What was the difference in the dot stimuli between tasks associated to the circles? \\
\hline 6
\end{tabular} $\begin{array}{l}\text { For some participants the amounts of dots changed after replying, so that one of } \\
\text { every pair of circles was associated with a task in which dots were added to the } \\
\text { correct square and removed from the other square (making the difference more } \\
\text { noticeable). The other circle was associated with an opposite task in which dots } \\
\text { were removed from the correct square and added to the other. Did it seem like this } \\
\text { was the case for you? Y/N }\end{array}$ \\
\hline \hline
\end{tabular}

Table 3. Debriefing questionnaire. This questionnaire, adapted from Kool et al. (2010) was administered at the end of each experiment to assess participants' awareness of the experimental manipulation. Note that question 6 varied slightly between experiments to represent the specific manipulation used.

Questions 1 and 3 were intended to probe conscious behavioral strategies. Although most participants reported selecting randomly, or based on cues' physical attributes such as color or location at first, many participants developed a conscious choice strategy or preference that did not rely only on cues' attributes $(60 \%, 90 \%, 75 \%, 75 \%, 58 \%$ of participants based on responses to questions 1 and 3 for Experiments 1-5 respectively). Reported strategies included error monitoring, perceived differences in difficulty, differences in confidence or a combination of these (e.g. "I started with a random colour and kept using that colour until I started making mistakes, became less sure about responses or missed a trial"). When asked 
explicitly if they developed a task preference, most participants responded positively $(65 \%$, $80 \%, 75 \%, 80 \%, 68 \%$ of participants replied yes to question 2 for Experiments $1-5$ respectively). Similarly, many participants reported finding one task easier (question 4: 60\%, $75 \%, 85 \%, 55 \%, 68 \%$ of participants respectively). To test if participants who reported experiencing differences in task difficulty were more likely to develop a preference for the high-confidence tasks, participants were grouped according to responses to question 4 (yes vs. no) and their task selections (high-confidence task selections $>50 \%$ vs high-confidence task selections $<50 \%$ ). Combining the data from Experiments $1-4,75 \%$ of participants who reported finding one task easier also demonstrated a preference for high-confidence tasks, vs. $64 \%$ of participants who did not report finding one task easier demonstrating a high-confidence preference. However, the relationship between perceived differences in difficulty and task choice was not significant $\left(\chi^{2}<1\right)$, suggesting that the demonstrated preference for highconfidence associated tasks was not driven by perceived differences in difficulty (though these results might also reflect a lack of sensitivity in our questionnaire and analysis).

When asked to explain differences between the tasks in terms of differences in the dot stimuli (question 5) responses varied, with participants referring to differences in dot numbers, stimulus refresh rate, stimulus display time, size and layout of dots, or simply stating they did not know ("I can't really tell which is why I think the difference was mainly in my head"). Only one participant in all the experiments combined explicitly mentioned post-decisional evidence manipulation, and even when the manipulation itself was explained (question 6), most participants did not identify the manipulation as being used in their case $(5 \%, 45 \%, 35 \%, 15 \%$, and $26 \%$ of participants answered "yes" to question 6 in Experiments 1-5, respectively). To ensure the preference effects found were not driven by participants who were explicitly aware of the evidence manipulation during the experiment, participants who responded yes to question 7 were removed from the analysis $(1,5,4,2,3$ participants $-5 \%, 25 \%, 20 \%, 10 \%$, 
8\% - for Experiments 1-5 respectively) and remaining participants' high-confidence tasks choice rates were compared to chance level. Results in Experiments 1, 3 and 4 remained significant $(t>2, \mathrm{p}<.05)$. In Experiment 2 results were not significant, most likely due to the large number of participants removed, but the trend remained $(t(14)=2.02, p=.06)$. In Experiment 5 the results remained non-significant $(\mathrm{t}<1)$.

In summary, the questionnaire results suggest that most participants used selection strategies that were based on metacognitive cues such as error monitoring, difficulty or confidence. Furthermore, they provide evidence that the preference we found does not depend on awareness to the evidence manipulation. However, it is important to note that the questionnaire lacks the sensitivity to provide insight into the relationship between general metacognitive awareness to differences between the tasks and the development of a preference, due to several limitations. First, to avoid biasing the way participants perform the task, the questionnaire was administered at the end of the experiment, rather than after every block. Thus, we could not assess the relationship between developing a preference and accurately identifying tasks differences (e.g. selecting the high-confidence cue more often on a specific block and reporting that it was associated with a preferable task because it was easier, led to higher confidence or induced less errors). Second, the wording of some questions might have led participants to give specific responses. For example, asking if one task was easier might have encouraged participants to report experiencing differences in task difficulties regardless of their experience during the experiment. They then might try and think what could lead to such differences in difficulty in response to question 5 , rather than reporting differences they noticed during the experiment. Thus, although the questionnaire responses provide useful insights regarding participants' strategies and metacognitive processes during the task, we are cautious in drawing any strong conclusions from these observations. 


\section{General Discussion}

The present study examined the role of decision confidence in task selection. A series of five experiments tested the hypothesis that confidence acts as an intrinsic cost-benefit factor, biasing people towards selection of tasks in which they experience higher confidence. To this end, we employed a task-selection paradigm in which the two competing tasks were identical except for the strength of post-decisional evidence, enabling us to study the effects of subjective confidence independent of objective performance. We found that participants consistently exhibit a bias towards selecting tasks in which they experience higher confidence. Moreover, we found that participants do not only utilize confidence as an internal errordetection system, avoiding tasks in which they believe or know they have made errors, but rather use it in a graded fashion so that they tend to select tasks in which they experience higher confidence in their correct responses. This preference was apparent when controlling for objective accuracy and the strength of evidence presented, and even when external feedback was provided to render internal error-monitoring of decisions unnecessary, indicating the consistent impact of confidence on task choice.

The notion that people tend to avoid effort has been prevalent in behavioral research for many years. Historically, the "law of least effort" (Hull, 1943) has been studied mainly in relation to physical demand, but recent advances have also provided empirical evidence for a bias against cognitively demanding tasks (Kool et al., 2010; Westbrook, Kester, \& Braver, 2013). Importantly, what exactly constitutes cognitive effort and what factors lead to its avoidance (for example, the degree to which behavior is guided by the subjective experience of effort, as opposed to objective performance), remain unclear (Shenhav et al., 2017). Our results contribute to a growing body of evidence providing support for the involvement of metacognitive awareness in task preferences. Moreover, since there has been substantial progress in specifying confidence in computational terms (Pleskac \& Busemeyer, 2010; Pouget 
et al., 2016; Sanders et al., 2016), linking confidence to choice behavior is particularly meaningful in formalizing a computational framework of task preferences.

Although several other studies have examined the role of metacognitive evaluations in task preferences, none have specifically addressed this question with relation to confidence. Previous investigations have studied the influence of perceived task difficulty (Desender et al., 2017) and perceived effort (Dunn et al., 2016). Our findings are in line with evidence from these studies, indicating that metacognitive cues play an important role in task selection. This congruency of effects raises the question of whether the task preference displayed by participants in our study was a result of their confidence or the result of other metacognitive cues, such as perceived task difficulty or effort.

Although it is likely that participants in general base their task selections on a broad metacognitive evaluation that incorporates multiple cues (Dunn et al., 2016), our manipulations and data favor an interpretation of our specific results as being driven primarily by decision confidence. First, except for post-decisional evidence, the tasks were similar in all aspects including participants' accuracy and response times. It is not clear why it should be more difficult or effortful to process post-decisional evidence across tasks, given that this evidence was not required for task performance. In contrast, the normative and computational basis for the influence of post-decisional evidence on confidence is well documented (Moran et al., 2015; Pleskac \& Busemeyer, 2010). Second, even allowing for the possibility that postdecisional evidence strength could somehow influence perceived effort or difficulty of an earlier perceptual decision, our regression analyses demonstrate that participants' confidence ratings account for unique variance above and beyond the evidence presented both pre- and post-decision. Indeed, in several cases, confidence was the only reliable predictor of participants' task selections from trial to trial. Thus, confidence is the most likely source of the emerging task preferences in our study. An interesting possibility to consider however, is that 
confidence acted on task choices by influencing perceived task difficulty. A recent study has demonstrated that response time, response repetition and response conflict all act as cues to subjective task difficulty (Desender, Van Opstal, \& Van Den Bussche, 2017). Since confidence usually correlates with response time and accuracy on tasks, it seems plausible that participants also incorporate confidence into their evaluation of task difficulty. In this case, confidence might have influenced task choices with perceived task difficulty acting as a mediator (although we did not find evidence for this suggestion in our debriefing questionnaire, but this might be due to a lack of sensitivity).

Many participants in our study reported experiencing differences in difficulty between the tasks, or developing strategies based on metacognitive evaluations such as error monitoring or decision confidence, but the majority of participants did not report awareness of the task manipulation. Only one participant identified post-decisional evidence differences between the tasks independently. Indeed, even when prompted with a description of the manipulation, most participants did not identify it as representing the experiment they had completed. Moreover, in Experiments 1, 3 and 4, we saw evidence for confidence preferences even when excluding participants who, after reading this description, reported being explicitly aware of the manipulation during the task. Thus, our results are not in line with recent studies indicating that cognitive effort affects task preferences only when people are aware of differences in cognitive demand (Desender et al., 2017; Gold et al., 2015), even if they are not aware of the source of these differences (Desender et al., 2017).

Across experiments, the preference for high confidence tasks tended to emerge relatively early in each block. However, participants did not display a persistent bias, but rather switched between the tasks throughout the blocks of the experiment, with confidence judgments predicting switches of task selections on a trial-by-trial basis. Thus, when considering the dynamics of the preference we found, it is likely that, rather than only 
developing a fixed and stable preference for one task driven by a general metacognitive awareness of task demands, participants also used confidence as an online monitoring system allowing them to adjust their behavior dynamically in a strategic fashion.

In all five experiments, we found that participants were more likely to repeat their task choice after trials with decisions they judged to be correct (vs. incorrect), but that gradations in decision confidence modulated task selection even beyond this binary classification that has been well-explored in the context of reinforcement learning: Thus, our participants were more likely to repeat task choices after they made correct responses with high vs. low confidence. Notably, however, we did not find a corresponding influence of confidence on task selection following trials reported as incorrect: participants were not more likely to repeat their task choice when they were more confident. Indeed, the numerical trend was in the opposite direction in Experiments 1, 4 and 5 (albeit only reaching statistical significance in the regression performed for the first experiment), with participants more likely to repeat when they were more certain they had made a mistake. Caution is appropriate in interpreting these weak and inconsistent results, but we suspect they reflect an interaction between two competing influences on task choice following errors - a bias away from tasks associated with errors (as detected internally or signaled via feedback), and a bias away from tasks associated with uncertainty about performance (or, relatedly, weak evidence).

This reasoning is relevant to the results of Experiment 5, in which the post-decisional evidence manipulation created exactly this difference across tasks - one in which more errors were detected vs. one in which weaker evidence was presented and more uncertainty experienced-and in which no consistent task preferences were apparent. There were, nevertheless, idiosyncratic preferences apparent in individual participants in Experiment 5 (Figure 6B), that were stronger than can be explained by chance variation: Task selections below .45 or greater than .55 have a binomial probability $<.05$ across our 320 -trial experiment, 
by which criterion 7 participants showed a clear preference for the task associated with higher confidence (boost incorrect task, weak evidence with fewer errors detected) and 10 preferred the task associated with stronger evidence (lower confidence, more errors detected). Thus, while it remains possible that an overall task preference was not detected due to an underpowered design (evidence was manipulated only after error trials, resulting in a smaller subset of manipulated trials compared to the previous experiments), the data suggest that this null finding is the result of individual differences in task preferences. Exploring individual differences in aversion to errors vs. uncertainty might be a useful avenue for future research, offering potential insights into differences in learning strategies, such as individual differences in information seeking and exploration vs. exploitation behaviors.

The importance of being able to monitor performance in an on-line way is intuitive, especially when external feedback is lacking. Indeed, in the influential framework proposed by Nelson \& Narens (1990), the function of metacognition is viewed in terms of control of behavior, whereby we monitor in order to control. With this in mind, perhaps the most intriguing result of our study is the emergence of a preference for high-confidence tasks even when external feedback is present and objective accuracy is well-matched (Experiment 4). In such conditions, the usefulness of internal monitoring resulting in a high confidence bias seems less evident. We suggest that, given the strong association between subjective confidence and objective performance, this preference is adaptive as fine-grained confidence is sometimes likely to act as a better predictor of future performance than trial accuracy (for example, consider a two-alternative task in which you are guessing, and know that you are - in this case, knowing that your guess happened to be correct vs. incorrect may not usefully inform future behavior). Indeed, as discussed above, we find that people do use confidence in a more sensitive way than simple error detection. This is further supported by results from Experiments 2 and 3, where a preference for a task associated with higher confidence emerged even when 
minimizing error monitoring differences. We conclude that confidence is used as a metacognitive cue that guides behavior even when feedback is present, and in a more sensitive manner than binary error monitoring. This suggestion is congruent with a recent study demonstrating that confidence is automatically integrated into brain activity in ventromedial prefrontal cortex, an area associated with encoding subjective value (Lebreton et al., 2015). Taken together with recent evidence that confidence can act as a reinforcement signal for perceptual learning when external feedback is absent (Guggenmos, Wilbertz, Hebart, \& Sterzer, 2016), our results suggest that confidence might play an important role in learning even when external feedback is present.

Collectively, our findings add to the growing body of evidence on the role of confidence in adaptive decision making. Previous studies have demonstrated that confidence plays a role in various contexts: linking performance across sequential decisions (van den Berg et al., 2016), guiding adaptive information seeking (Desender et al., 2018, 2019), preparing for upcoming choices according to anticipated difficulty (Boldt, Schiffer, Waszak, \& Yeung, 2019), balancing the ecological imperatives of exploration vs. exploitation in value-based choice (Boldt, Blundell, \& De Martino, 2019) and arbitrating among competing opinions across individuals (Bahrami et al., 2010; Bang et al., 2014). Our findings are particularly congruent with recent evidence indicating that confidence is represented on a common scale across tasks of different modalities, and can be compared between tasks as efficiently and precisely as within the same task (de Gardelle et al., 2016; de Gardelle \& Mamassian, 2014). De Gardelle and colleagues suggest that confidence is used as a common currency for judging our decisions, allowing different tasks to be compared and prioritized. Their results complement our findings that confidence biases decision making when all else is equal. By combining the conclusions from these studies, we can speculate that confidence is used as a general cue that guides behavior and task preferences, even when comparing between different 
tasks. Future studies might extend this investigation to consider the effects of confidence preferences across different tasks and modalities. More broadly, when taken with evidence that confidence can guide behavior flexibly across multiple contexts, the present findings support a conception whereby metacognitive processes support a variety of strategic behaviors of different kinds and spanning a range of timescales, based on a common computation (specifically here the subjective evaluation of decision accuracy).

In conclusion, the present results add to our understanding of the functional role of confidence in decision making. We find that people show a preference for situations in which they are more confident, even when controlling for other plausible influences on task selection. Since confidence and performance are strongly related, this preference can be useful for adaptive decision making in our daily life, guiding us towards tasks in which we are ultimately more likely to succeed. These results fit well with the concept of metacognition as a system in which monitoring is performed in order to enable subsequent control and adjustment of behavior. 


\section{References}

Apps, M. A. J., Grima, L. L., Manohar, S., \& Husain, M. (2015). The role of cognitive effort in subjective reward devaluation and risky decision-making. Scientific Reports, 5, 16880. https://doi.org/10.1038/srep16880

Bahrami, B., Olsen, K., Latham, P. E., Roepstorff, A., Rees, G., \& Frith, C. D. (2010). Optimally Interacting Minds. Science, 329(5995), 1081-1085. https://doi.org/10.1126/science.1185718

Bang, D., Fusaroli, R., Tylén, K., Olsen, K., Latham, P. E., Lau, J. Y. F., ... Bahrami, B. (2014). Does interaction matter? Testing whether a confidence heuristic can replace interaction in collective decision-making. Consciousness and Cognition, 26(1), 13-23. https://doi.org/10.1016/j.concog.2014.02.002

Baranski, J. V., \& Petrusic, W. M. (1994). The calibration and resolution of confidence in perceptual judgments. Perception \& Psychophysics, 55(4), 412-428. https://doi.org/10.3758/BF03205299

Bjork, R. A., Dunlosky, J., \& Kornell, N. (2013). Self-Regulated Learning: Beliefs, Techniques, and Illusions. Annual Review of Psychology, 64(1), 417-444. https://doi.org/10.1146/annurev-psych-113011-143823

Boldt, A., Blundell, C., \& De Martino, B. (2019). Confidence modulates exploration and exploitation in value-based learning. Neuroscience of Consciousness, 2019(1), 1-12. https://doi.org/10.1093/nc/niz004

Boldt, A., Schiffer, A.-M., Waszak, F., \& Yeung, N. (2019). Confidence Predictions Affect Performance Confidence and Neural Preparation in Perceptual Decision Making. Scientific Reports, 9(1), 4031. https://doi.org/10.1038/s41598-019-40681-9

Boldt, A., \& Yeung, N. (2015). Shared neural markers of decision confidence and error detection. The Journal of Neuroscience: The Official Journal of the Society for 
Neuroscience, 35(8), 3478-3484. https://doi.org/10.1523/JNEUROSCI.0797-14.2015

Brainard, D. H. (1997). The Psychophysics Toolbox. Spatial Vision, 10(4), 433-436.

Retrieved from http://www.ncbi.nlm.nih.gov/pubmed/9176952

Charles, L., \& Yeung, N. (2019). Dynamic sources of evidence supporting confidence judgments and error detection. Journal of Experimental Psychology: Human Perception and Performance, 45(1), 39-52. https://doi.org/10.1037/xhp0000583

de Gardelle, V., Le Corre, F., \& Mamassian, P. (2016). Confidence as a Common Currency between Vision and Audition. Plos One, 11(1), e0147901. https://doi.org/10.1371/journal.pone.0147901

de Gardelle, V., \& Mamassian, P. (2014). Does Confidence Use a Common Currency Across Two Visual Tasks? Psychological Science, 25(6), 1286-1288. https://doi.org/10.1177/0956797614528956

Desender, K., Boldt, A., \& Yeung, N. (2018). Subjective Confidence Predicts Information Seeking in Decision Making. Psychological Science. https://doi.org/10.1177/0956797617744771

Desender, K., Calderon, C. B., Van Opstal, F., \& Van den Bussche, E. (2017). Avoiding the Confict: Metacognitive Awareness Drives the Selection of Low-Demand Context. Journal of Experimental Psychology: Human Perception and Performance, 43(7), 1397-1410. https://doi.org/https://doi.org/10.1037/xhp0000391

Desender, K., Murphy, P., Boldt, A., Verguts, T., \& Yeung, N. (2019). A post-decisional neural marker of confidence predicts information-seeking in decision-making. The Journal of Neuroscience, 39(17), 2620-18. https://doi.org/10.1523/JNEUROSCI.262018.2019

Desender, K., Van Opstal, F., \& Van Den Bussche, E. (2017). Subjective experience of difficulty depends on multiple cues. Scientific Reports, 7(March), 1-14. 
https://doi.org/10.1038/srep44222

Dunn, T. L., Lutes, D. J. C., \& Risko, E. F. (2016). Metacognitive evaluation in the avoidance of demand. Journal of Experimental Psychology: Human Perception and Performance, 42(9), 1372-1387. https://doi.org/http://dx.doi.org/10.1037/xhp0000236

Faul, F., Erdfelder, E., Lang, A.-G., \& Buchner, A. (2007). G*Power: A flexible statistical power analysis program for the social, behavioral, and biomedical sciences. Behavior Research Methods, 39(2), 175-191. https://doi.org/10.3758/BF03193146

Flavell, J. H. (1979). Metacognition and cognitive monitoring: A new area of cognitivedevelopmental inquiry. American Psychologist, 34(10), 906-911. https://doi.org/10.1037/0003-066x.34.10.906

Fleming, S. M., \& Daw, N. D. (2017). Self-evaluation of decision-making: A general bayesian framework for metacognitive computation. Psychological Review, 124(1), 91114. https://doi.org/10.1037/rev0000045

Fleming, S. M., \& Frith, C. D. (2014). The Cognitive Neuroscience of Metacognition. (S. M. Fleming \& C. D. Frith, Eds.), The Cognitive Neuroscience of Metacognition (Vol. 9783642451). Berlin, Heidelberg: Springer Berlin Heidelberg. https://doi.org/10.1007/978-3-642-45190-4

Fleming, S. M., Van Der Putten, E. J., \& Daw, N. D. (2018). Neural mediators of changes of mind about perceptual decisions. Nature Neuroscience, 21(4), 617-624. https://doi.org/10.1038/s41593-018-0104-6

Gold, J. M., Kool, W., Botvinick, M. M., Hubzin, L., August, S., \& Waltz, J. a. (2015). Cognitive effort avoidance and detection in people with schizophrenia. Cognitive, Affective \& Behavioral Neuroscience, 15(1), 145-154. https://doi.org/10.3758/s13415014-0308-5

Guggenmos, M., Wilbertz, G., Hebart, M. N., \& Sterzer, P. (2016). Mesolimbic confidence 
signals guide perceptual learning in the absence of external feedback, 1-19. https://doi.org/10.7554/eLife.13388

Hajcak, G., \& Foti, D. (2008). Errors are aversive: Defensive motivation and the error-related negativity: Research report. Psychological Science, 19(2), 103-108. https://doi.org/10.1111/j.1467-9280.2008.02053.x

Holroyd, C. B., \& Coles, M. G. H. (2002). The neural basis of human error processing: Reinforcement learning, dopamine, and the error-related negativity. Psychological Review, 109(4), 679-709. https://doi.org/10.1037/0033-295X.109.4.679

Holroyd, C. B., Yeung, N., Coles, M. G. H., \& Cohen, J. D. (2005). A mechanism for error detection in speeded response time tasks. Journal of Experimental Psychology: General, 134(2), 163-191. https://doi.org/10.1037/0096-3445.134.2.163

Hull, C. L. (1943). Principles of behavior : an introduction to behavior theory. New York; London : Appleton-Century.

Kahneman, D., \& Tversky, A. (1984). Choices, values, and frames. American Psychologist, 39(4), 341. https://doi.org/10.1037/0003-066X.39.4.341

Kepecs, A., Uchida, N., Zariwala, H. a, \& Mainen, Z. F. (2008). Neural correlates, computation and behavioural impact of decision confidence. Nature, 455(7210), $227-$ 231. https://doi.org/10.1038/nature07200

Kiani, R., \& Shadlen, M. N. (2009). Representation of confidence associated with a decision by neurons in the parietal cortex. Science (New York, N.Y.), 324(2009), 759-764. https://doi.org/10.1126/science.1169405

Kleiner, M., Brainard, D., Pelli, D., Ingling, A., Murray, R., \& Broussard, C. (2007). What's new in psychtoolbox-3. Perception, 36(14), 1-16.

Kool, W., McG uire, J. T., Rosen, Z. B., \& Botvinick, M. M. (2010). Decision making and the avoidance of cognitive demand. J Exp Psychol Gen, 139(4), 665-682. 
https://doi.org/10.1037/a0020198

Koriat, A. (2006). Metacognition and consciousness. The Cambridge Handbook of Consciousness, 3(2), 289-326.

https://doi.org/http://dx.doi.org/10.1017/CBO9780511816789.012

Koriat, A., \& Levy-Sadot, R. (2001). The combined contributions of the cue-familiarity and accessibility heuristics to feelings of knowing. Journal of Experimental Psychology. Learning, Memory, and Cognition, 27(1), 34-53. Retrieved from http://www.ncbi.nlm.nih.gov/pubmed/11204106

Lebreton, M., Abitbol, R., Daunizeau, J., \& Pessiglione, M. (2015). Automatic integration of confidence in the brain valuation signal. Nature Neuroscience, 18(8), 1159-1167. https://doi.org/10.1038/nn.4064

Moran, R., Teodorescu, A. R., \& Usher, M. (2015). Post choice information integration as a causal determinant of confidence: Novel data and a computational account. Cognitive Psychology, 78, 99-147. https://doi.org/10.1016/j.cogpsych.2015.01.002

Murayama, K., Blake, A. B., Kerr, T., \& Castel, A. D. (2016). When enough is not enough: Information overload and metacognitive decisions to stop studying information. Journal of Experimental Psychology. Learning, Memory, and Cognition, 42(6), 914-924. https://doi.org/10.1037/xlm0000213

Murphy, P. R., Robertson, I. H., Harty, S., \& O’Connell, R. G. (2015). Neural evidence accumulation persists after choice to inform metacognitive judgments. ELife, 4(DECEMBER2015), 1-23. https://doi.org/10.7554/eLife.11946

Nelson, T. O., Dunlosky, J., Graf, A., \& Narens, L. (1994). Utilization of metacognitive judgments in the allocation of study during multitrial learning. Psychological Science, 5(4), 207-213. https://doi.org/10.1111/j.1467-9280.1994.tb00502.x

Nelson, T. O., \& Narens, L. (1990). Metamemory: A Theoretical Framework and New 
Findings. The Psychology of Learning and Motivation, 26, 125-173.

https://doi.org/10.1016/S0079-7421(08)60053-5

Pelli, D. G. (1997). The VideoToolbox software for visual psychophysics: transforming numbers into movies. Spatial Vision, 10(4), 437-442. Retrieved from http://www.ncbi.nlm.nih.gov/pubmed/9176953

Pleskac, T. J., \& Busemeyer, J. R. (2010). Two-stage dynamic signal detection: a theory of choice, decision time, and confidence. Psychological Review, 117(3), 864-901. https://doi.org/10.1037/a0019737

Pouget, A., Drugowitsch, J., \& Kepecs, A. (2016). Confidence and certainty: distinct probabilistic quantities for different goals. Nature Neuroscience, 19(3), 366-374. https://doi.org/10.1038/nn.4240

Rabbitt, P. M. (1966). Errors and error correction in choice-response tasks. Experimental Psychology, 71(2), 264-272. https://doi.org/10.1037/h0022853

Reder, L. M. (1987). Strategy selection in question answering. Cognitive Psychology, 19(1), 90-138. https://doi.org/10.1016/0010-0285(87)90005-3

Sanders, J. I., Hangya, B., \& Kepecs, A. (2016). Signatures of a Statistical Computation in the Human Sense of Confidence. Neuron, 90(3), 499-506.

https://doi.org/10.1016/j.neuron.2016.03.025

Shenhav, A., Musslick, S., Lieder, F., Kool, W., Griffiths, T. L., Cohen, J. D., \& Botvinick, M. M. (2017). Toward a Rational and Mechanistic Account of Mental Effort. Annual Review of Neuroscience, 40(1), 99-124. https://doi.org/10.1146/annurev-neuro-072116031526

Solomon, R. L. (1948). The influence of work on behavior. Psychological Bulletin, 45(1), 140. https://doi.org/10.1037/h0055527

van den Berg, R., Zylberberg, A., Kiani, R., Shadlen, M. N., \& Wolpert, D. M. (2016). 
Confidence Is the Bridge between Multi-stage Decisions. Current Biology, 26(23), 3157-3168. https://doi.org/10.1016/j.cub.2016.10.021

Walton, M. E., Kennerley, S. W., Bannerman, D. M., Phillips, P. E. M., \& Rushworth, M. F. S. (2006). Weighing up the benefits of work: Behavioral and neural analyses of effortrelated decision making. Neural Networks, 19(8), 1302-1314. https://doi.org/10.1016/j.neunet.2006.03.005

Westbrook, A., Kester, D., \& Braver, T. S. (2013). What Is the Subjective Cost of Cognitive Effort? Load, Trait, and Aging Effects Revealed by Economic Preference. PLoS ONE, 8(7), 1-8. https://doi.org/10.1371/journal.pone.0068210

Westbrook, A., Lamichhane, B., \& Braver, T. (2019). The Subjective Value of Cognitive Effort is Encoded by a Domain-General Valuation Network. The Journal of Neuroscience, 39(20), 3071-18. https://doi.org/10.1523/JNEUROSCI.3071-18.2019

Yeung, N., \& Summerfield, C. (2012). Metacognition in human decision-making: confidence and error monitoring. Philosophical Transactions of the Royal Society of London. Series B, Biological Sciences, 367(1594), 1310-1321. https://doi.org/10.1098/rstb.2011.0416

Yeung, N., \& Summerfield, C. (2014). Shared mechanisms for confidence judgements and error detection in human decision making. In S. M. Fleming \& C. D. Frith (Eds.), The Cognitive Neuroscience of Metacognition (Vol. 9783642451, pp. 147-167). Berlin, Heidelberg: Springer Berlin Heidelberg. https://doi.org/10.1007/978-3-642-45190-4_7

Yu, S., Pleskac, T. J., \& Zeigenfuse, M. D. (2015). Dynamics of postdecisional processing of confidence. Journal of Experimental Psychology: General, 144(2), 489-510. https://doi.org/10.1037/xge0000062 